\title{
Stress-driven instability in growing multilayer films
}

\author{
Zhi-Feng Huang* and Rashmi C. Desai ${ }^{\dagger}$ \\ Department of Physics, University of Toronto, Toronto, Ontario, Canada M5S 1A7
}

(Dated: December 11, 2002 (revised); accepted for publication in Phys. Rev. B)

\begin{abstract}
We investigate the stress-driven morphological instability of epitaxially growing multilayer films, which are coherent and dislocation-free. We construct a direct elastic analysis, from which we determine the elastic state of the system recursively in terms of that of the old states of the buried layers. In turn, we use the result for the elastic state to derive the morphological evolution equation of surface profile to first order of perturbations, with the solution explicitly expressed by the growth conditions and material parameters of all the deposited layers. We apply these results to two kinds of multilayer structures. One is the alternating tensile/compressive multilayer structure, for which we determine the effective stability properties, including the effect of varying surface mobility in different layers, its interplay with the global misfit of the multilayer film, and the influence of asymmetric structure of compressive and tensile layers on the system stability. The nature of the asymmetry properties found in stability diagrams is in agreement with experimental observations. The other multilayer structure that we study is one composed of stacked strained/spacer layers. We also calculate the kinetic critical thickness for the onset of morphological instability and obtain its reduction and saturation as number of deposited layers increases, which is consistent with recent experimental results. Compared to the single-layer film growth, the behavior of kinetic critical thickness shows deviations for upper strained layers.
\end{abstract}

\section{INTRODUCTION}

In recent years, much attention has been focused on the heteroepitaxial growth of multilayer films for the purpose of fabricating high-quality low-dimensional structures. ${ }^{1}$ Currently, two kinds of multilayers are being actively studied: (i) the alternating tensile/compressive multilayers exploited as multiple quantum wells or short-period superlattices; ${ }^{2-8}$ and (ii) multisheets of coherently strained layers (or islands) alternately separated by spacer layers of substrate materials (strained/spacer arrays). ${ }^{1,9-18}$ Experimentally, these multilayer structures are grown coherently on the substrates, without the generation of undesirable misfit dislocations. When instability occurs and develops during the multilayer films growth, one of the most important phenomena is the formation of self-organized nanostructures. For the tensile/compressive multilayers, lateral modulations have been found during the epitaxial growth, showing as the modulated pattern of alternating vertical or columnar structures with strong contrast compositions. ${ }^{2-7}$ These modulation structures that form spontaneously are very useful for the quantum wires fabrication. For the strained/spacer multilayer structure, a remarkable phenomenon is the vertical self-organization of multisheet arrays of three-dimensional (3D) islands (quantum dots). ${ }^{1,9-17}$ The islands are vertically correlated in successive strained layers, with the increasing uniformity (including island size and density) as the number of layers increases. These low-dimensional nanostructures (such as quantum wires and quantum dots), realized through the development of multilayer film instability, exhibit novel electronic and optical properties, including the band structure, carrier transport, etc., and are of intense interests both for fundamental studies and for application to devices with enhanced optoelectronic performance.

The mechanism of occurrence of instability in strained-layer growth is believed to be stress-driven. When a singlelayer strained film (with single component) is grown epitaxially on a substrate, the morphological instability develops due to the nonuniform strain energy distribution with respect to any surface corrugations and the surface diffusion processes driven by the difference of local strain energy. ${ }^{19,20}$ When other layers composed of different materials, and consequently with different misfit strains with respect to the substrate, are deposited subsequently, as in the multilayer growth, the coupling of strain fields caused by buried layers and the growing one determines the morphological evolution of surface profile. This coupling is the source for self-organized growth of vertical islands array in the strained/spacer multilayer. ${ }^{9,11}$ For the tensile/compressive multilayer films, the phenomenon of lateral composition modulations can be explained by the morphological instability caused by the coupling strains, leading to the surface undulations and then to both the layer thickness modulations and composition modulations in the overall film through the stacking of layers. ${ }^{6,7}$

Although in the coherent multilayer systems the processes of morphological evolution are mainly dominated by elastic effects, our understanding of the mechanisms is incomplete and not well developed due to the complexity of the multilayer structures. One of the main difficulties of the theoretical investigations is in determining the elastic fields of the system, especially the surface strain field caused by all the buried layers or islands. First, the system is inhomogeneous due to the stacking of layers with different materials; second, the geometry of this system, which is composed of top surface, finite thickness of film layers and the semi-infinite substrate, is not symmetric and is 
therefore different from the case of bulk materials. Moreover, the interfaces between different layers are generally not planar (except for the interfaces between strained and thick enough spacer layers), and then the system cannot be simplified to an equivalent and solvable semi-infinite solid as in the study of single-layer films. ${ }^{20-22}$ Thus, it is very difficult to exactly calculate the elastic fields of the system.

The theoretical work for multilayer systems is rather sparse, and some approximations have to be used. For the tensile/compressive multilayer structure, Sridhar et al. ${ }^{23}$ considered an infinite periodic and symmetric system with the dynamics controlled by interfacial diffusion, and focused on the interior layers that are far away from both surface and substrate, instead of the surface morphology that we are interested in this paper. In the recent work of Shilkrot et $a l .,{ }^{24}$ the surface diffusion and morphological evolution is discussed, with the determination of stability for multilayer films. The Eshelby procedure ${ }^{25}$ is used for each buried interface, which is assumed to separately contribute to surface strain. Then the problem is approximated to be that of a misfit inclusion in a semi-infinite homogeneous isotropic elastic medium with the presence of a free surface. For a system of stacked coherent strained islands (strained/spacer multilayer), in the work of Tersoff et al. ${ }^{9}$ each buried island is approximately treated as a point defect, and its contribution to surface strain is determined by using the continuum elasticity solutions of Maradudin and Wallis. ${ }^{26}$ Their results explain the vertical correlation between islands of successive layers, showing the self-organization of the system. The size and shape of stacked islands also exhibit the self-organized effects, as studied in a later work by Liu et al.. ${ }^{17}$

Here, we propose a recursive procedure and develop a continuum, dynamical model to directly describe the morphological evolution of these coherent multilayer structures during the epitaxial growth. Using the isotropic linear elasticity theory, we calculate in section II the elastic fields of the multilayer system directly from the linearized boundary conditions at all the buried interfaces and the top surface. Based on our elastic analysis, the coupling and influence of all the buried layers and interfaces are determined in section III to describe the evolution of surface morphological perturbation. Within this framework, we investigate the stability properties of multilayer growing films by performing a linear analysis. Also, the effects of different material parameters and growth conditions on morphological stability/instability of multilayer films are determined and discussed.

We first apply in section IV these results to the alternating tensile/compressive multilayer system, determining the effective stability properties. We investigate the effect of different surface mobilities of strained layers on the stability results, and the interplay between the relative mobility and the global misfit of multilayer film (especially the asymmetry between globally tensile and compressive films). In the absence of global strain, i.e., with a global strain-balanced condition, we find an interesting property that the system is more stable for the asymmetric structure (with respect to layer thickness and misfit) of compressive and tensile layers. The effects of relative surface tension and growth temperature are also studied.

Then in section $\mathrm{V}$ we investigate the strained/spacer multilayer structure (for the case of flat surface of spacer layers), and calculate the kinetic critical thickness for the onset of 3D instability. We obtain a reduction and saturation of critical thickness in the upper strained layers, well reproducing the recent experimental observations. We also find that the behavior of kinetic critical thickness for upper strained layers deviates from that of single-layer film growth for large misfit and small deposition rate.

\section{MODEL AND ELASTIC FORMULATION}

Assume that a multilayer film is coherently deposited on a semi-infinite substrate (denoted by index 0 ) and consists of different layers $i=1,2, \ldots, k$. In the general case, material of the deposited layer $i$ can be different from that of the substrate and that of all the other layers, and its misfit with respect to the substrate is denoted as $\epsilon_{i}$. As we know from the heteroepitaxial growth of a single-layer film, ${ }^{19-22,27,28}$ the nonzero misfit strain can be relieved through the development of morphological instability, leading to a nonplanar surface profile $z=h_{1}(x, y)=l_{1}+\sum_{\mathbf{q}} \hat{h}_{1}(\mathbf{q}) \exp \left(i q_{x} x+\right.$ $\left.i q_{y} y\right)$ for the first layer 1 , with a coherent interface at $z=h_{0}=\zeta(x, y)=\sum_{\mathbf{q}} \hat{\zeta}(\mathbf{q}) \exp \left(i q_{x} x+i q_{y} y\right)$ (the average position $\bar{\zeta}=0$ ) between film and substrate, where $l_{1}$ is the average thickness of first layer and $\hat{h}_{1}(\hat{\zeta})$ denotes the surface (interface) morphological perturbation. Here we only consider the morphological modulations of the film, corresponding to the deposition of only a single component in each layer, and do not consider the compositional evolution which is important in alloy growth systems and which results in more complicated properties due to its coupling with the morphological evolution. ${ }^{21,22,27,28}$

When the second and higher layers are deposited with different materials and thus with different misfits, the undulating surface is buried as a frozen rough interface if the growth temperature $T$ is not too high making interlayer diffusion negligible. Then the elastic state of the system is altered as a result of new deposition, and the morphological evolution of the new free surface is different from that of the first single layer due to the coupling of strain fields in different layers and the influence of buried interfaces on the elastic field and energy of the multilayer film as a whole. For 
a $k$-layer system, the growing surface profile is represented as $h_{k}(x, y, t)=\sum_{i=1}^{k-1} l_{i}+v_{k} t+\sum_{\mathbf{q}} \hat{h}_{k}(\mathbf{q}, t) \exp \left(i q_{x} x+i q_{y} y\right)$, with $l_{i}(1 \leq i<k)$ the average thickness of $i$ th layer underneath, $v_{k}$ the deposition rate of top layer $k$, and $\hat{h}_{k}$ the top surface perturbation. When we consider the atomic diffusion along the top surface and the uniform deposition of material as the dominant mass-transport processes, the evolution equation of the surface morphological profile is given by

$$
\frac{\partial h_{k}}{\partial t}=\Gamma_{k} \sqrt{g_{k}} \nabla_{s}^{2} \frac{\delta \mathcal{F}}{\delta h_{k}}+v_{k}+\sqrt{g_{k}} \nabla \cdot \boldsymbol{\eta}_{c}+\eta_{k},
$$

where $g_{k}=1+\left|\nabla h_{k}\right|^{2}$ denotes the determinant of surface metric for top layer $k$, and $\Gamma_{k}=D_{s, k} N_{s, k} / k_{B} T N_{v, k}^{2}$ is the surface mobility with $D_{s, k}$ the surface diffusivity, $k_{B}$ the Boltzmann constant, $N_{s, k}$ and $N_{v, k}$ respectively the number density of atoms per unit surface area and per unit volume of layer $k$. Both the conserved thermal noise $\boldsymbol{\eta}_{c}$ and nonconserved deposition noise $\eta_{k}$ are included in the above evolution equation, but are neglected in the following studies since they do not alter the results of linear analysis presented here. In Eq. (1), the free energy $\mathcal{F}$ of the system consists of two terms:

$$
\mathcal{F}=\gamma_{k} \int d^{2} r \sqrt{g_{k}}+\int_{-\infty}^{h_{k}} d^{3} r \mathcal{E}
$$

The first term is the surface energy with $\gamma_{k}$ the surface tension at the top free surface of layer $k$, and tends to stabilize the film morphology. $\mathcal{E}$ in the second term represents the strain energy density, and is, in linear elasticity theory, $\mathcal{E}=S_{\alpha \beta \xi \rho} \sigma_{\alpha \beta} \sigma_{\xi \rho} / 2(\alpha, \beta, \xi, \rho=x, y, z)$, where $\sigma_{\alpha \beta}$ is the stress tensor. For an elastically isotropic system that we assume here, the elastic compliance tensor $S_{\alpha \beta \xi \rho}=\delta_{\alpha \xi} \delta_{\beta \rho}(1+\nu) / E-\delta_{\alpha \beta} \delta_{\xi \rho} \nu / E$ with Young's modulus $E$ and Poisson ratio $\nu$. The form of total free energy in Eq. (2) implies that we have neglected the wetting effect of each deposited layer, which is not dominant when the layer thickness is assumed to be large enough. The wetting effect is related to the interaction between two successive layers or between film and substrate, and is important for thin films with thickness less than 3 ML. ${ }^{29}$

Substituting Eq. (2) into Eq. (1), we obtain the expression for surface profile evolution of top growing layer $k$ :

$$
\partial h_{k} / \partial t=\Gamma_{k} \sqrt{g_{k}} \nabla_{s}^{2}\left[\gamma_{k} \kappa+\mathcal{E}_{k}\left(h_{k}, h_{k-1}, \ldots, \zeta\right)\right]+v_{k},
$$

where $\kappa$ is the surface curvature, and $\mathcal{E}_{k}$ is the elastic energy density evaluated at surface $z=h_{k}$ and is dependent on all the surface and interface morphologies due to the coupling of strain fields. Since here we focus on the stability of the multilayer system and hence the early evolution regime with small perturbations, to determine the corresponding important properties we only need the first-order evolution equation for surface morphology. Expanding Eq. (3) in Fourier space to first order of the morphological perturbation, we obtain the linearized evolution equation that will be used in the following linear analysis:

$$
\partial \hat{h}_{k}(\mathbf{q}, t) / \partial t=-\Gamma_{k} q^{2}\left[\gamma_{k} q^{2} \hat{h}_{k}(\mathbf{q}, t)+\hat{\mathcal{E}}_{k}(\mathbf{q})\right]
$$

with $\hat{\mathcal{E}}_{k}(\mathbf{q})$ the first order elastic energy density evaluated at the growing surface position. For the buried interfaces between layers $i+1$ and $i(1 \leq i \leq k-1)$, the morphology $h_{i}(x, y)=\sum_{j=1}^{i} l_{j}+\sum_{\mathbf{q}} \hat{h}_{i}(\mathbf{q}) \exp \left(i q_{x} x+i q_{y} y\right)$ as well as its perturbation $\hat{h}_{i}$ are also determined by Eqs. (3) and (4) during the growth of layer $i$, with the parameters of layer $k$ replaced by those of layer $i$; then the morphology at the top of the $i$ th layer remains unchanged (with the evaluation at $t=l_{i} / v_{i}$ ) when the subsequent layers are deposited.

The above evolution form of the surface morphology is analogous to that used for single-layer film growth, ${ }^{20-22,27,28}$ but the crucial difference is in the determination of elastic energy density $\mathcal{E}$, which is affected by all the underlying layers and the substrate, which are made up of different materials, in the inhomogeneous multilayer systems. To obtain the elastic free energy in linear elasticity theory, the stress tensor of each layer $i \quad(1 \leq i \leq k$ or substrate $i=0$ with $\left.\epsilon_{0}=0\right)$

$$
\sigma_{\alpha \beta, i}=2 \mu_{i}\left[\frac{\nu_{i}}{1-2 \nu_{i}} u_{\rho \rho, i} \delta_{\alpha \beta}+u_{\alpha \beta, i}-\frac{1+\nu_{i}}{1-2 \nu_{i}} \epsilon_{i} \delta_{\alpha \beta}\right]
$$

$(\alpha, \beta=x, y, z)$ should be evaluated. Here $\mu_{i}=E_{i} / 2\left(1+\nu_{i}\right)$ is the shear modulus of layer $i$, with $E_{i}$ and $\nu_{i}$ the Young's modulus and Poisson ratio, and $u_{\alpha \beta, i}=\left(\partial_{\alpha} u_{\beta, i}+\partial_{\beta} u_{\alpha, i}\right) / 2$ is the linear elastic strain tensor governed by the elastic displacement field $u_{\alpha, i}$ in layer $i$.

The ideal way to determine the elastic state of the system is to directly solve the mechanical equilibrium equation

$$
\partial_{\beta} \sigma_{\alpha \beta, i}=0
$$


and then obtain the elastic fields $u_{\alpha, i}$ for all the coupling layers and substrate $(0 \leq i \leq k)$ without any approximations. Here we have assumed that in this nonequilibrium system of growing multilayer film, the mechanical equilibrium (6) occurs instantaneously compared to the film morphological evolution process. The corresponding boundary conditions can be obtained by the consideration of the negligible external pressure on top growing surface, leading to

$$
\sigma_{\alpha \beta, k} n_{\beta, k}=0 \quad \text { at } \quad z=h_{k}(x, y, t),
$$

and the coherence at the buried $(i+1) / i$ interface for $0 \leq i \leq k-1$ :

$$
\sigma_{\alpha \beta, i+1} n_{\beta, i}=\sigma_{\alpha \beta, i} n_{\beta, i} \quad \text { and } \quad u_{\alpha, i+1}=u_{\alpha, i} \quad \text { at } \quad z=h_{i}(x, y),
$$

with $n_{\beta, k}$ and $n_{\beta, i}$ the unit vectors normal to the surface of layer $k$ and $(i+1) / i$ interface, respectively. Also, in the semi-infinite substrate, the decay of strain field far away from the film results in

$$
u_{\alpha, 0} \rightarrow 0 \quad \text { for } \quad z \rightarrow-\infty \text {. }
$$

Therefore, for a $k$-layers system with one top surface and $k$ interfaces, we have to solve $6(k+1)$ equations from boundary conditions (7)-(9) to determine the solution of mechanical equilibrium equation (6) for all the layers and substrate. Also, due to the nonplanar interfaces between different layers, even if the elastic constants of all the layers and substrate are assumed to be identical, the stability results of the system here are not equivalent to those of a simplified semi-infinite solid as in the former study of single layer film with flat film-substrate interface. ${ }^{20-22}$ So to obtain the exact and direct solution of these equations is very difficult for large $k$, even in a linear analysis.

Here we propose a recursive procedure to directly calculate the strain fields of this multilayer system to first order of perturbations. In the framework of isotropic elasticity and the linear analysis, the elastic free energy can be determined from the exact solution of the (first-order) elastic fields and then used in dynamical equation (4) for the analysis of morphological evolution.

The basic state of this multilayer structure corresponds to a uniform film with a planar growing surface at constant rate $v_{k}$ and all the planar and coherent interfaces underneath. Since the basic state of the substrate is stress-free, with $\bar{u}_{\alpha, 0}=\bar{u}_{\alpha \beta, 0}=\bar{\sigma}_{\alpha \beta, 0}=0(\alpha, \beta=x, y, z)$, and all the surface and interfaces are flat, due to the coherency of the film there are no in-plane displacement and strain for all the layers, so that $\bar{u}_{x, i}=\bar{u}_{y, i}=0$ and $\bar{u}_{x \beta, i}=\bar{u}_{y \beta, i}=0$ ( $\beta=x, y, z$ and $1 \leq i \leq k)$. Also, from the boundary condition (7) on the top surface and the coherency at all the flat interfaces, we have $\bar{\sigma}_{\alpha z, i}=0$ with $\alpha=x, y, z$ in each layer $i$. The displacement in the $z$ direction is nonzero: $\bar{u}_{z, i}=\bar{u}_{i}\left(z-\sum_{j=1}^{i-1} l_{j}\right)+\sum_{j=1}^{i-1} \bar{u}_{j} l_{j}$ with $\bar{u}_{i}=\epsilon_{i}\left(1+\nu_{i}\right) /\left(1-\nu_{i}\right)$, and the Poisson relaxation in the $z$ direction leads to constant strain $\bar{u}_{z z, i}=\bar{u}_{i}$. The basic-state film is stressed in the lateral direction, with stresses $\bar{\sigma}_{x x, i}=\bar{\sigma}_{y y, i}=\bar{\sigma}_{i}=-2 \mu_{i} \bar{u}_{i}$ for all the layers. Note that in general case, the elastic constants used here $\left(E_{i}, \nu_{i}\right.$, and $\left.\mu_{i}\right)$ can be different in different layers and substrate.

The expansion form of the mechanical equilibrium equation for each layer or substrate can be obtained by substituting Eq. (5) into Eq. (6) and expanding in Fourier space with respect to perturbations around the above basic state (in the following expressions all the hatted quantities denote the corresponding Fourier perturbations):

$$
\left(1-2 \nu_{i}\right)\left(\partial_{z}^{2}-q^{2}\right)\left[\begin{array}{c}
\hat{u}_{x, i} \\
\hat{u}_{y, i} \\
\hat{u}_{z, i}
\end{array}\right]+\left[\begin{array}{c}
i q_{x} \\
i q_{y} \\
\partial_{z}
\end{array}\right]\left(i q_{x} \hat{u}_{x, i}+i q_{y} \hat{u}_{y, i}+\partial_{z} \hat{u}_{z, i}\right)=0
$$

for $i$ th layer $(1 \leq i \leq k)$ or substrate $(i=0)$, with general solution

$$
\begin{aligned}
{\left[\begin{array}{c}
\hat{u}_{x, i} \\
\hat{u}_{y, i} \\
\hat{u}_{z, i}
\end{array}\right]=\left[\begin{array}{c}
\alpha_{x, i} \\
\alpha_{y, i} \\
\alpha_{z, i}
\end{array}\right] \cosh (q z)+\left[\begin{array}{c}
\beta_{x, i} \\
\beta_{y, i} \\
\beta_{z, i}
\end{array}\right] \sinh (q z) } \\
-\left[\begin{array}{c}
C_{i} i q_{x} / q \\
C_{i} i q_{y} / q \\
D_{i}
\end{array}\right] z \sinh (q z)-\left[\begin{array}{c}
D_{i} i q_{x} / q \\
D_{i} i q_{y} / q \\
C_{i}
\end{array}\right] z \cosh (q z)
\end{aligned}
$$

for $1 \leq i \leq k$, and

$$
\left[\begin{array}{l}
\hat{u}_{x, 0} \\
\hat{u}_{y, 0} \\
\hat{u}_{z, 0}
\end{array}\right]=\left[\begin{array}{l}
\alpha_{x, 0} \\
\alpha_{y, 0} \\
\alpha_{z, 0}
\end{array}\right] e^{q z}-\left[\begin{array}{c}
i q_{x} / q \\
i q_{y} / q \\
1
\end{array}\right] B z e^{q z}
$$

for substrate $i=0$ due to boundary condition (9), where the parameters $C_{i}=\left(i q_{x} \alpha_{x, i}+i q_{y} \alpha_{y, i}+q \beta_{z, i}\right) /\left(3-4 \nu_{i}\right)$, $D_{i}=\left(i q_{x} \beta_{x, i}+i q_{y} \beta_{y, i}+q \alpha_{z, i}\right) /\left(3-4 \nu_{i}\right)$, and $B=\left(i q_{x} \alpha_{x, 0}+i q_{y} \alpha_{y, 0}+q \alpha_{z, 0}\right) /\left(3-4 \nu_{0}\right)$. These forms of differential 
equations and general solutions are the same as those for single-layer film, ${ }^{20}$ but with different linearized boundary conditions. From Eq. (7), we have

$$
\hat{\sigma}_{\beta z, k}=i q_{\beta} \bar{\sigma}_{k} \hat{h}_{k} \quad(\beta=x, y) \quad \text { and } \quad \hat{\sigma}_{z z, k}=0,
$$

at top surface $z=\bar{h}_{k}=\sum_{i=1}^{k-1} l_{i}+v_{k} t$, while at the interface between layers $i+1$ and $i$, i.e., at $z=\bar{h}_{i}=\sum_{j=1}^{i} l_{j}$ $(0 \leq i \leq k-1)$, boundary condition (8) leads to

$$
\begin{aligned}
& -i q_{\beta}\left(\bar{\sigma}_{i+1}-\bar{\sigma}_{i}\right) \hat{h}_{i}+\hat{\sigma}_{\beta z, i+1}=\hat{\sigma}_{\beta z, i} \quad(\beta=x, y), \\
& \hat{\sigma}_{z z, i+1}=\hat{\sigma}_{z z, i},
\end{aligned}
$$

and

$$
\begin{aligned}
& \hat{u}_{\beta, i+1}=\hat{u}_{\beta, i} \quad(\beta=x, y), \\
& \left(\bar{u}_{i+1}-\bar{u}_{i}\right) \hat{h}_{i}+\hat{u}_{z, i+1}=\hat{u}_{z, i} .
\end{aligned}
$$

Note that when $i=0$, we have $\bar{u}_{0}=\bar{\sigma}_{0}=0$ and $\hat{h}_{0}=\hat{\zeta}$ for the substrate, and condition (9) has already been used to obtain the solution form (12).

All the above mechanical equilibrium equations and boundary conditions are for the $k$-layer system, and are also fulfilled by the $(k-1)$-layer system before layer $k$ is deposited, with $k$ replaced by $k-1$. Let us denote the elastic displacement field for this $(k-1)$-layer system (with average film thickness $\sum_{i=1}^{k-1} l_{i}$ ) by $\left.u_{\alpha, i}\right|_{k-1}$ with $0 \leq i \leq k-1$ and $\alpha=x, y, z$, where " $\left.\right|_{k-1}$ " represents that the system is of $(k-1)$ layers. When a new layer $k$ is deposited, the elastic state of the whole multilayer is changed and the displacement field is now $\left.u_{\alpha, i}\right|_{k}$ (for the $k$-layer system with average film thickness $\left.\sum_{i=1}^{k-1} l_{i}+v_{k} t\right)$. For all the buried $(k-1)$ layers and substrate, this elastic field is composed of two contributions:

$$
\left.u_{\alpha, i}\right|_{k}=\left.u_{\alpha, i}^{o}\right|_{k}+\left.u_{\alpha, i}^{n}\right|_{k} \quad \text { for } \quad 0 \leq i \leq k-1 .
$$

Here the first contribution $\left.u_{\alpha, i}^{o}\right|_{k}$ is the old-state field before the deposition of new layer $k$, and is identical to $\left.u_{\alpha, i}\right|_{k-1}$ determined by the previous $(k-1)$-layer system and assumed to be known. The second contribution $\left.u_{\alpha, i}^{n}\right|_{k}$ represents all the change of strain field caused by the new deposition, and is the unknown new-state field. The other unknown quantity is the elastic field of the new top layer $k:\left.u_{\alpha, k}\right|_{k}$, and both of them should be calculated from the mechanical equilibrium equations and boundary conditions.

Since the old-state field $\left.u_{\alpha, i}^{o}\right|_{k}=\left.u_{\alpha, i}\right|_{k-1}$ obeys the mechanical equilibrium equation (6) (or (10)), from Eq. (15) we know that new-state elastic field $\left.u_{\alpha, i}^{n}\right|_{k}$ also follows the same equation, with the same solution forms (11) and (12), and so does the unknown elastic field $\left.u_{\alpha, k}\right|_{k}$ of the new layer $k$. For the linearized boundary conditions (13) and (14), when we replace $\hat{u}_{\alpha, i}$ by formula $(15), \hat{\sigma}_{\alpha \beta, i}$ by $\left.\hat{\sigma}_{\alpha \beta, i}\right|_{k}=\left.\hat{\sigma}_{\alpha \beta, i}^{o}\right|_{k}+\left.\hat{\sigma}_{\alpha \beta, i}^{n}\right|_{k}(0 \leq i \leq k-1)$ which can be obtained according to Eq. (5), and use the similar boundary conditions for $(k-1)$-layer system, which are: at $z=\bar{h}_{k-1}$,

$$
\left.\hat{\sigma}_{\beta z, k-1}\right|_{k-1}=i q_{\beta} \bar{\sigma}_{k-1} \hat{h}_{k-1} \quad \text { and }\left.\quad \hat{\sigma}_{z z, k-1}\right|_{k-1}=0 \text {, }
$$

and at $z=\bar{h}_{i}$ for $(i+1) / i$ interface $(0 \leq i \leq k-2)$,

$$
\begin{gathered}
-i q_{\beta}\left(\bar{\sigma}_{i+1}-\bar{\sigma}_{i}\right) \hat{h}_{i}+\left.\hat{\sigma}_{\beta z, i+1}\right|_{k-1}=\left.\hat{\sigma}_{\beta z, i}\right|_{k-1} \quad \text { and }\left.\hat{\sigma}_{z z, i+1}\right|_{k-1}=\left.\hat{\sigma}_{z z, i}\right|_{k-1}, \\
\left.\hat{u}_{\beta, i+1}\right|_{k-1}=\left.\hat{u}_{\beta, i}\right|_{k-1} \text { and }\left(\bar{u}_{i+1}-\bar{u}_{i}\right) \hat{h}_{i}+\left.\hat{u}_{z, i+1}\right|_{k-1}=\left.\hat{u}_{z, i}\right|_{k-1},
\end{gathered}
$$

we can derive the simplified boundary conditions for new-state fields $\left.u_{\alpha, i}^{n}\right|_{k}(0 \leq i \leq k-1)$ and for the top-layer field $\left.u_{\alpha, k}\right|_{k}$. On top surface of the $k$-layer system, i.e., $z=\bar{h}_{k}$, condition (13) remains unchanged:

$$
\left.\hat{\sigma}_{\beta z, k}\right|_{k}=i q_{\beta} \bar{\sigma}_{k} \hat{h}_{k} \quad(\beta=x, y) \quad \text { and }\left.\quad \hat{\sigma}_{z z, k}\right|_{k}=0
$$

while at $z=\bar{h}_{k-1}$ for the $k /(k-1)$ interface, we have (with $\beta=x, y$ and $\left.u_{\alpha, k-1}\right|_{k-1}=\left.u_{\alpha, k-1}^{o}\right|_{k}$ for $\alpha=x, y, z$ )

$$
\begin{gathered}
-i q_{\beta} \bar{\sigma}_{k} \hat{h}_{k-1}+\left.\hat{\sigma}_{\beta z, k}\right|_{k}=\left.\hat{\sigma}_{\beta z, k-1}^{n}\right|_{k} \quad \text { and }\left.\quad \hat{\sigma}_{z z, k}\right|_{k}=\left.\hat{\sigma}_{z z, k-1}^{n}\right|_{k} \\
\left.\hat{u}_{\beta, k}\right|_{k}=\left.\hat{u}_{\beta, k-1}\right|_{k-1}+\left.\hat{u}_{\beta, k-1}^{n}\right|_{k} \quad \text { and } \\
\left(\bar{u}_{k}-\bar{u}_{k-1}\right) \hat{h}_{k-1}+\left.\hat{u}_{z, k}\right|_{k}=\left.\hat{u}_{z, k-1}\right|_{k-1}+\left.\hat{u}_{z, k-1}^{n}\right|_{k} .
\end{gathered}
$$


At all the other underlying interfaces, the boundary conditions are much simpler:

$$
\left.\hat{\sigma}_{\alpha z, i+1}^{n}\right|_{k}=\left.\hat{\sigma}_{\alpha z, i}^{n}\right|_{k} \quad \text { and }\left.\quad \hat{u}_{\alpha, i+1}^{n}\right|_{k}=\left.\hat{u}_{\alpha, i}^{n}\right|_{k}
$$

at $z=\bar{h}_{i}$ with $\alpha=x, y, z$ and $0 \leq i \leq k-2$.

Note that the boundary conditions (18) for all the interfaces buried below layer $k-1$ are equivalent to those of planar interfaces in the elastic media with displacement fields $\left.\hat{u}_{\alpha, i}^{n}\right|_{k}$. Thus, from all the boundary conditions (16)-(18) and the mechanical equilibrium equations followed by elastic fields $\left.\hat{u}_{\alpha, k}\right|_{k}$ and $\left.\hat{u}_{\alpha, i}^{n}\right|_{k}$, we can obtain an effective elastic system for which the elastic state is identical (to first order) to that of the $k$-layer system we are interested in. This effective system is composed of a growing and undulating surface of top layer $k$ with condition (16), a nonplanar interface between layers $k$ and $k-1$ with special boundary conditions (17), as well as the effective planar interfaces (with respect to the new-state fields $\left.\hat{u}_{\alpha, i}^{n}\right|_{k}$ ) between all the other buried layers and between film and substrate. The schematic illustration of this procedure is shown in Fig. 1 (a) and (b), where part (a) presents the original $k$-layer system with nonplanar surface and interfaces and with elastic fields $\left.u_{\alpha, i}\right|_{k}(0 \leq i \leq k)$ to be determined, and part (b) is the effective system (related to elastic fields $\left.u_{\alpha, k}\right|_{k}$ for top layer $k$ and $\left.u_{\alpha, i}^{n}\right|_{k}$ for all layers underneath) that we obtain here to first order of perturbations.

This elastic system can be further simplified and then solved exactly when we consider the identical elastic constants for all the layers and substrate. The assumption of identical elastic constants is applicable to the system of which substrate and all the layers in the film are composed of materials with similar elastic constants, and is used in the following calculations so that we could focus on the effects related to surface mobilities and layer thicknesses. Note that for a single-layer film, grown on a flat substrate, the elastic behavior of the system is equivalent to that of a semi-infinite stressed solid in the case of equal elastic constants. ${ }^{20,22}$ This can also be obtained by combining the elastic solution forms in Eq. (11) for layer 1 and in Eq. (12) for substrate with the flat boundary condition (18), which leads to the identical (new-state) elastic fields for the first layer and substrate, i.e., $\left.\hat{u}_{\alpha, 1}^{n}\right|_{k}=\left.\hat{u}_{\alpha, 0}^{n}\right|_{k}$. Similarly, from all the effectively planar boundaries below layer $k-1$ (as shown in Eq. (18) and Fig. 1 (b)), we can obtain the same solution for the new-state elastic fields in all the buried layers and substrate: $\left.\hat{u}_{\alpha, 0}^{n}\right|_{k}=\left.\hat{u}_{\alpha, 1}^{n}\right|_{k}=\ldots=\left.\hat{u}_{\alpha, k-1}^{n}\right|_{k}$. This implies that the elastic state (with respect to $\left.\hat{u}_{\alpha, i}^{n}\right|_{k}$ ) for all these layers and substrate $(0 \leq i \leq k-1)$ is equivalent to that of a single semi-infinite effective medium.

Consequently, under the approximation of identical elastic constants and in a first order analysis, we can simplify the original $k$-layer structure to an elastically equivalent system composed of a single layer $k$ (with elastic field $\left.u_{\alpha, k}\right|_{k}$ ) and an underlying effective semi-infinite elastic medium (with respect to field $\left.u_{\alpha, k-1}^{n}\right|_{k}$ ), as illustrated in Fig. 1 (c). Now we only have one nonplanar interface at $z=h_{k-1}$, but with special boundary condition (17) which is different from that of the usual single-layer film growth. The undulating free surface at $z=h_{k}$ satisfies the condition (16) as usual. The elastic state of this effective system can be solved directly, in a manner similar to that for the case of a single-layer film with rough film-substrate interface (but with different boundary condition). ${ }^{28}$ Thus, from Eq. (15) (and noting the boundary condition (17)), we can obtain the elastic fields of the original $k$-layer system $\left.\hat{u}_{\alpha, i}\right|_{k}$ $(0 \leq i \leq k)$ expressed by that of the previously grown $(k-1)$-layer system $\left.\hat{u}_{\alpha, j}\right|_{k-1}(0 \leq j \leq k-1)$.

Using the same method, elastic fields $\left.\hat{u}_{\alpha, j}\right|_{k-1}$ can be calculated in terms of $\left.\hat{u}_{\alpha, j}\right|_{k-2}$ for the $(k-2)$-layer system. This procedure is repeated by expressing the elastic fields for $i$-layer system in terms of those for $(i-1)$-layer system with $i=k, k-1, k-2, \ldots, 2$, until we reach the single-layer system for which the elastic fields $\left.\hat{u}_{\alpha, i}\right|_{1}(i=0,1)$ have already been obtained for both the cases of $\operatorname{planar}^{20}$ and rough $^{28}$ film-substrate interface. Thus, from this recursive procedure we can derive the first-order elastic fields of the $k$-layer growing system in the expression of known material parameters, and then calculate the elastic free energy for the determination of morphological evolution equation.

\section{MORPHOLOGICAL EVOLUTION FOR GENERAL CASE}

In the linearized equation (4) for surface morphological evolution, the first-order elastic energy density is $\hat{\mathcal{E}}_{k}(\mathbf{q})=$ $\bar{\sigma}_{k}\left(\left.\hat{u}_{x x}\right|_{k}+\left.\hat{u}_{y y}\right|_{k}\right)$ with strain tensors $\left.\hat{u}_{x x}\right|_{k}$ and $\left.\hat{u}_{y y}\right|_{k}$ evaluated at top surface $z=\bar{h}_{k}$. For the case of identical elastic constants $(E, \mu$, and $\nu)$ in all the layers and the substrate, the method in Sec. II (or the effective system in Fig. 1 (c)) yields

$$
\begin{aligned}
\hat{\mathcal{E}}_{k}(\mathbf{q}) & =-E^{\prime} \epsilon_{k}^{2} q \hat{h}_{k}+E^{\prime}\left[\epsilon_{k}^{2}-\frac{\epsilon_{k} \epsilon_{k-1}}{2(1-\nu)} q l_{k}\right] e^{-q l_{k}} q \hat{h}_{k-1} \\
& +\frac{E}{1-\nu} \epsilon_{k}\left[\left(q l_{k}-1\right) e^{-q l_{k}}\left(\left.i q_{x} \hat{u}_{x, k-1}\right|_{k-1}+\left.i q_{y} \hat{u}_{y, k-1}\right|_{k-1}\right)_{z=\bar{h}_{k-1}}\right. \\
& \left.-q l_{k} e^{-q l_{k}}\left(\left.q \hat{u}_{z, k-1}\right|_{k-1}\right)_{z=\bar{h}_{k-1}}\right],
\end{aligned}
$$


where $E^{\prime}=2 E(1+\nu) /(1-\nu)$ and $l_{k}=v_{k} t$ is defined for the top growing layer $k$. Also $\left.\hat{u}_{\alpha, k-1}\right|_{k-1}\left(z=\bar{h}_{k-1}\right)$ is obtained from the solution of elastic fields according to the recursive procedure presented in Sec. II, with the result:

$$
\begin{aligned}
&\left(\left.i q_{x} \hat{u}_{x, k-1}\right|_{k-1}+\left.i q_{y} \hat{u}_{y, k-1}\right|_{k-1}\right)_{z=\bar{h}_{k-1}}=2(1-\nu)\left[q \bar{u}_{k-1} \hat{h}_{k-1}\right. \\
&\left.-q \sum_{j=1}^{k-2}\left(\bar{u}_{j+1}-\bar{u}_{j}\right) e^{-q \sum_{i=j+1}^{k-1} l_{i}} \hat{h}_{j}-q \bar{u}_{1} e^{-q \sum_{i=1}^{k-1} l_{i}} \hat{\zeta}\right] \\
&\left(\left.i q_{x} \hat{u}_{x, k-1}\right|_{k-1}+\left.i q_{y} \hat{u}_{y, k-1}\right|_{k-1}-\left.q \hat{u}_{z, k-1}\right|_{k-1}\right)_{z=\bar{h}_{k-1}}=q \bar{u}_{k-1} \hat{h}_{k-1}
\end{aligned}
$$

with $\bar{u}_{j}=\epsilon_{j}(1+\nu) /(1-\nu)$.

Substituting Eq. (19) into Eq. (4), we obtain the evolution equation of surface morphological perturbation:

$$
\begin{aligned}
\partial \hat{h}_{k}(q, t) / \partial t & =\sigma_{k} \hat{h}_{k}(q, t)-\Gamma_{k} E^{\prime} \epsilon_{k} q^{3} e^{-q v_{k} t} \\
& \times\left[\sum_{j=1}^{k-1}\left(\epsilon_{j+1}-\epsilon_{j}\right) e^{-q \sum_{i=j+1}^{k-1} l_{i}} \hat{h}_{j}(q)+\epsilon_{1} e^{-q \sum_{i=1}^{k-1} l_{i}} \hat{\zeta}(q)\right]
\end{aligned}
$$

where

$$
\sigma_{k}=\Gamma_{k}\left(E^{\prime} \epsilon_{k}^{2} q^{3}-\gamma_{k} q^{4}\right)
$$

corresponds to the (single-layer) perturbation growth rate without the coupling of buried layers and interfaces, and the interface perturbations $\hat{h}_{j}$ and $\hat{\zeta}$ are time independent due to the absence of interdiffusion. In Eq. (20), the influence of elastic properties and morphologies of all buried layers on the evolution of the top surface profile is shown explicitly. Note that due to the exponential decay term $\exp \left(-q \sum_{i=j+1}^{k-1} l_{i}\right)$, there is much smaller influence from lower buried layers, as usually expected.

Note that the above linearized dynamical equation for surface morphology is different from that in Ref. 24 by Shilkrot et al. for a periodic structure composed of alternating layers of materials $A$ and $B$ (see Eq. (10) there), which was also derived to first order of perturbations with the assumption of equal elastic constants within the multilayer structure. This is attributed to the different methods in the elastic analysis of the system. Shilkrot et al. used the Eshelby procedure ${ }^{25}$ by assuming each buried interface as the interface of a misfitting inclusion in a semi-infinite homogeneous and isotropic elastic medium with free surface, and considered the contribution from each interface to be separate. Here we apply a more direct procedure to determine the elastic state, and find that for this highly inhomogeneous system, an underlying interface can only be treated as one between an effective semi-infinite homogeneous medium (with respect to a new-state elastic field $u_{\alpha}^{n}$ ) and another elastic medium (of top layer) with finite thickness and free top surface, under the condition of identical elastic constants and linear analysis (see Fig. 1 (c)). The explicit form of our evolution equation is simpler than that of Ref. 24 and so is our solution as well as the corresponding recurrence relation as shown in Sec. IV for periodic $A / B$ system.

Using the initial condition $\hat{h}_{k}(q, t=0)=\hat{h}_{k-1}(q)$, we can easily solve the evolution equation (20), with the result for the top surface morphology:

$$
\begin{aligned}
\hat{h}_{k}(q, t) & =\frac{1}{q v_{k}+\sigma_{k}}\left\{\left[\left(q v_{k}+\Gamma_{k}\left(E^{\prime} \epsilon_{k} \epsilon_{k-1} q^{3}-\gamma_{k} q^{4}\right)\right) e^{\sigma_{k} t}+\Gamma_{k} E^{\prime} \epsilon_{k}\left(\epsilon_{k}-\epsilon_{k-1}\right) q^{3} e^{-q v_{k} t}\right] \hat{h}_{k-1}\right. \\
& \left.+\left(e^{-q v_{k} t}-e^{\sigma_{k} t}\right) \Gamma_{k} E^{\prime} q^{3} \epsilon_{k}\left[\sum_{j=1}^{k-2}\left(\epsilon_{j+1}-\epsilon_{j}\right) e^{-q \sum_{i=j+1}^{k-1} l_{i}} \hat{h}_{j}-\epsilon_{1} e^{-q \sum_{i=1}^{k-1} l_{i}} \hat{\zeta}\right]\right\}
\end{aligned}
$$

which is one of the central results of this paper. In Eq. $(22)$, the morphologies of buried interfaces $\left(\hat{h}_{j}, j=1, \ldots\right.$, $k-1$ ) remain unchanged during the evolution process of top layer, as a result of negligible bulk diffusion of the film, and can be determined from the similar calculation for lower $j$-layer system. In the case of nonzero misfits for all the deposited layers, (i.e., $\epsilon_{i} \neq 0$ for all $i=1,2, \ldots, k$ ), Eq. (22) can be reduced recursively through the expression of the surface or interface perturbation in terms of the perturbations of two lower successive interfaces, leading to the form

$$
\left(\begin{array}{c}
\hat{h}_{k}(q, t) \\
\hat{h}_{k-1}(q)
\end{array}\right)=\mathbf{L}_{k} \prod_{j=3}^{k-1} \overline{\mathbf{L}}_{j}\left(\begin{array}{c}
\hat{h}_{2}(q) \\
\hat{h}_{1}(q)
\end{array}\right) .
$$


In Eq. (23), $\mathbf{L}_{k}$ and $\overline{\mathbf{L}}_{j}(0 \leq j \leq k-1)$ are $2 \times 2$ matrices:

$$
\mathbf{L}_{k}=\left(\begin{array}{cc}
L_{k, 1}(q, t) & L_{k, 2}(q, t) \\
1 & 0
\end{array}\right), \quad \overline{\mathbf{L}}_{j}=\left(\begin{array}{cc}
\bar{L}_{j, 1}(q) & \bar{L}_{j, 2}(q) \\
1 & 0
\end{array}\right)
$$

where

$$
\begin{aligned}
L_{k, 1}(q, t) & =\frac{1}{q v_{k}+\sigma_{k}}\left\{\left[q v_{k}+\Gamma_{k}\left(E^{\prime} \epsilon_{k} \epsilon_{k-1} q^{3}-\gamma_{k} q^{4}\right)\right] e^{\sigma_{k} t}+\Gamma_{k} E^{\prime} \epsilon_{k}\left(\epsilon_{k}-\epsilon_{k-1}\right) q^{3} e^{-q v_{k} t}\right\} \\
& -L_{k, 2}(q, t) e^{-\sigma_{k-1} l_{k-1} / v_{k-1}}, \\
L_{k, 2}(q, t) & =-\frac{q v_{k-1}+\sigma_{k-1}}{q v_{k}+\sigma_{k}} \frac{\Gamma_{k} \epsilon_{k}}{\Gamma_{k-1} \epsilon_{k-1}} \frac{e^{\sigma_{k-1} l_{k-1} / v_{k-1}-q l_{k-1}}}{e^{-q l_{k-1}}-e^{\sigma_{k-1} l_{k-1} / v_{k-1}}}\left(e^{-q v_{k} t}-e^{\sigma_{k} t}\right),
\end{aligned}
$$

and $\bar{L}_{j, 1}(q)$ and $\bar{L}_{j, 2}(q)$ are obtained by replacing $k$ with $j$ as well as $t$ with $l_{j} / v_{j}$ in $L_{k, 1}(q, t)$ and $L_{k, 2}(q, t)$, respectively. Results for first and second layer perturbations $\hat{h}_{1}$ and $\hat{h}_{2}$ are presented in the Appendix, for both rough $(\zeta \neq 0)$ and planar $(\zeta=0)$ film-substrate interfaces. Therefore, from Eqs. (23)-(25) and the results in the Appendix, we obtain the explicit expression for first-order surface morphological perturbation $\hat{h}_{k}(q, t)$ in terms of known material parameters, which can be directly used for stability analysis as shown in the following sections.

Note that the above solution results apply to the general case for which growth parameters (including the layer thicknesses, misfits, surface mobilities, surface tensions, and deposition rate) of different layers can be different, and then apply to both the non-periodic and periodic multilayer structures. Also we have assumed the identical elastic constants in the whole multilayer film, and the nonzero misfit strains for all the layers. In the case of zero misfit $\epsilon_{i}=0$ for some spacer layers $i$ (corresponding to the strained/spacer multilayer structures), the solution forms are simpler and will be discussed in Sec. V.

\section{TENSILE/COMPRESSIVE MULTILAYERS: PERIODIC $A / B$ SYSTEM}

Here we apply the results for morphological evolution, derived in the last section, to the periodic tensile/compressive multilayer structures that have been investigated in many experiments, such as $\operatorname{InAs} / \mathrm{AlAs}$ on $\operatorname{InP}(001),{ }^{2-5}$ GaInP(InGaAs)/InAsP on $\operatorname{InP}(001),{ }^{6-8}$ etc. This type of multilayer film consists of two kinds of alternating layers with materials $A$ and $B$. Then we have misfit strain $\epsilon_{A}$, layer thickness $l_{A}$, surface mobility $\Gamma_{A}$, surface tension $\gamma_{A}$, and deposition rate $v_{A}$ for odd-number strained layers, and the corresponding parameters $\epsilon_{B}, l_{B}, \Gamma_{B}, \gamma_{B}$, and $v_{B}$ for even-number layers, with nonzero misfits for all the layers: $\epsilon_{A} \neq 0$ and $\epsilon_{B} \neq 0$.

\section{A. Formulation of morphological evolution}

For this periodic $A / B$ system, the solution for the top surface morphological perturbation can be directly obtained from Eqs. (23)-(25). For a $k$-layer structure, when $k$ is odd implying that the top layer $k$ is of material $A$, we have

$$
\left(\begin{array}{l}
\hat{h}_{k}^{*}\left(q^{*}, \tau\right) \\
\hat{h}_{k-1}^{*}\left(q^{*}\right)
\end{array}\right)=\mathbf{L}_{A}\left(\overline{\mathbf{L}}_{B} \overline{\mathbf{L}}_{A}\right)^{\frac{k-3}{2}}\left(\begin{array}{c}
\hat{h}_{2}^{*}\left(q^{*}\right) \\
\hat{h}_{1}^{*}\left(q^{*}\right)
\end{array}\right) \quad(k \geq 3),
$$

with $2 \times 2$ matrices:

$$
\mathbf{L}_{A(B)}=\left(\begin{array}{cc}
L_{A(B) 1}\left(q^{*}, \tau\right) & L_{A(B) 2}\left(q^{*}, \tau\right) \\
1 & 0
\end{array}\right), \quad \overline{\mathbf{L}}_{A(B)}=\left(\begin{array}{cc}
\bar{L}_{A(B) 1}\left(q^{*}\right) & \bar{L}_{A(B) 2}\left(q^{*}\right) \\
1 & 0
\end{array}\right),
$$

while for even number of deposited layers, the top layer $k$ is of material $B$ and the solution form is

$$
\left(\begin{array}{c}
\hat{h}_{k}^{*}\left(q^{*}, \tau\right) \\
\hat{h}_{k-1}^{*}\left(q^{*}\right)
\end{array}\right)=\mathbf{L}_{B} \overline{\mathbf{L}}_{A}\left(\overline{\mathbf{L}}_{B} \overline{\mathbf{L}}_{A}\right)^{\frac{k-4}{2}}\left(\begin{array}{l}
\hat{h}_{2}^{*}\left(q^{*}\right) \\
\hat{h}_{1}^{*}\left(q^{*}\right)
\end{array}\right) \quad(k \geq 4) .
$$

Here, the solutions (26) and (28) are expressed in dimensionless form, and we have used the rescaling

$$
q^{*}=q l_{A}^{0}, \quad \tau=t / \tau_{A}^{0}, \quad \text { and } \quad \hat{h}_{i}^{*}=\hat{h}_{i} / l_{A}^{0} \quad(0 \leq i \leq k),
$$


with the characteristic length and time scale respectively given as:

$$
l_{A}^{0}=\gamma_{A} /\left(E^{\prime} \epsilon_{A}^{2}\right),
$$

and

$$
\tau_{A}^{0}=\gamma_{A}^{3} /\left(\Gamma_{A} E^{\prime 4} \epsilon_{A}^{8}\right) .
$$

These are determined according to the (single-layer film) growth of layer 1 (assumed of material $A$ ). We also introduce the characteristic velocity

$$
v_{A}^{0}=l_{A}^{0} / \tau_{A}^{0}=\Gamma_{A} E^{\prime 3} \epsilon_{A}^{6} / \gamma_{A}^{2},
$$

as well as the following transformations

$$
\begin{gathered}
l_{A}^{*}=l_{A} / l_{A}^{0}, \quad l_{B}^{*}=l_{B} / l_{A}^{0}, \\
v_{A}^{*}=v_{A} / v_{A}^{0}, \quad v_{B}^{*}=v_{B} / v_{A}^{0}, \\
\Gamma_{B}^{*}=\Gamma_{B} / \Gamma_{A}, \quad \epsilon_{B}^{*}=\epsilon_{B} / \epsilon_{A}, \quad \gamma_{B}^{*}=\gamma_{B} / \gamma_{A} .
\end{gathered}
$$

Then the matrix elements in Eq. (27) are rescaled as

$$
\begin{aligned}
& L_{A 1}\left(q^{*}, \tau\right)=\frac{1}{q^{*} v_{A}^{*}+\sigma_{A}^{*}}\left\{\left[q^{*} v_{A}^{*}+\left(\epsilon_{B}^{*} q^{* 3}-q^{* 4}\right)\right] e^{\sigma_{A}^{*} \tau}+\left(1-\epsilon_{B}^{*}\right) q^{* 3} e^{-q^{*} v_{A}^{*} \tau}\right\} \\
& -L_{A 2}\left(q^{*}, \tau\right) e^{-\left(\sigma_{B}^{*} / v_{B}^{*}\right) l_{B}^{*}}, \\
& L_{A 2}\left(q^{*}, \tau\right)=-\frac{q^{*}+\sigma_{B}^{*} / v_{B}^{*}}{q^{*} v_{A}^{*}+\sigma_{A}^{*}} \frac{1}{\left(\Gamma_{B}^{*} / v_{B}^{*}\right) \epsilon_{B}^{*}} \frac{e^{\left(\sigma_{B}^{*} / v_{B}^{*}\right) l_{B}^{*}-q^{*} l_{B}^{*}}}{e^{-q^{*} l_{B}^{*}-e^{\left(\sigma_{B}^{*} / v_{B}^{*}\right) l_{B}^{*}}}}\left(e^{-q^{*} v_{A}^{*} \tau}-e^{\sigma_{A}^{*} \tau}\right), \\
& L_{B 1}\left(q^{*}, \tau\right)=\frac{1}{q^{*}+\sigma_{B}^{*} / v_{B}^{*}}\left\{\left[q^{*}+\frac{\Gamma_{B}^{*}}{v_{B}^{*}}\left(\epsilon_{B}^{*} q^{* 3}-\gamma_{B}^{*} q^{* 4}\right)\right] e^{\sigma_{B}^{*} \tau}+\frac{\Gamma_{B}^{*}}{v_{B}^{*}} \epsilon_{B}^{*}\left(\epsilon_{B}^{*}-1\right) q^{* 3} e^{-q^{*} v_{B}^{*} \tau}\right\} \\
& \text { - } L_{B 2}\left(q^{*}, \tau\right) e^{-\sigma_{A}^{*} l_{A}^{*} / v_{A}^{*}} \text {, } \\
& L_{B 2}\left(q^{*}, \tau\right)=-\frac{q^{*} v_{A}^{*}+\sigma_{A}^{*}}{q^{*}+\sigma_{B}^{*} / v_{B}^{*}} \frac{\Gamma_{B}^{*}}{v_{B}^{*}} \epsilon_{B}^{*} \frac{e^{\sigma_{A}^{*} l_{A}^{*} / v_{A}^{*}-q^{*} l_{A}^{*}}}{e^{-q^{*} l_{A}^{*}-e^{\sigma_{A}^{*} l_{A}^{*} / v_{A}^{*}}}}\left(e^{-q^{*} v_{B}^{*} \tau}-e^{\sigma_{B}^{*} \tau}\right),
\end{aligned}
$$

with $\bar{L}_{A 1}\left(q^{*}\right), \bar{L}_{A 2}\left(q^{*}\right), \bar{L}_{B 1}\left(q^{*}\right)$, and $\bar{L}_{B 2}\left(q^{*}\right)$ obtained by replacing $\tau$ with $l_{A}^{*} / v_{A}^{*}$ or $l_{B}^{*} / v_{B}^{*}$ in Eq. (34), and

$$
\begin{aligned}
& \sigma_{A}^{*}=q^{* 3}-q^{* 4}, \\
& \sigma_{B}^{*}=\Gamma_{B}^{*}\left(\epsilon_{B}^{* 2} q^{* 3}-\gamma_{B}^{*} q^{* 4}\right) .
\end{aligned}
$$

¿From Eq. (35), we can have $\sigma_{A}^{*}>0$ or $\sigma_{B}^{*}>0$ for a certain wave number $q^{*}$, and then the expressions in Eq. (34) show that $\left|L_{A 1(2)}\right| \gg 1$ or $\left|L_{B 1(2)}\right| \gg 1$ when time $\tau$ is very large. Consequently, we can obtain from Eqs. (26) and (28) that for a certain top surface layer, when the growth time is large enough the amplitude of surface perturbation $\hat{h}$ will grow rapidly with time. Thus, in principle this multilayer system is unstable.

However, in real experiments the thickness of each layer $\left(l_{A}\right.$ or $\left.l_{B}\right)$ is fixed, including the top layer, then the growth of this multilayer system could be effectively stable or unstable. ${ }^{2-5,24}$ That is, when the film surface is flat after the growth of large number of strained layers $(k \gg 1)$, i.e., the initial perturbation decays with further deposition, the system is effectively stable; otherwise, if the initial perturbation is amplified and grows in amplitude, the system is unstable. For the latter case, the film surface is observed to be very rough, with regular or irregular modulations.

The stability properties can be calculated from Eqs. (26) and (28), where $\hat{h}_{k}^{*}\left(q^{*}, \tau\right), \mathbf{L}_{A}$, and $\mathbf{L}_{B}$ should be replaced by $\hat{h}_{k}^{*}\left(q^{*}\right), \overline{\mathbf{L}}_{A}$, and $\overline{\mathbf{L}}_{B}$, respectively, and then the top surface perturbation is mainly determined by the power of matrix $\overline{\mathbf{L}}=\overline{\mathbf{L}}_{B} \overline{\mathbf{L}}_{A}$, which has two eigenvalues $\lambda_{1}$ and $\lambda_{2}$ given by

$$
\lambda_{1,2}\left(q^{*}\right)=\frac{1}{2}\left\{\left(\bar{L}_{A 2}+\bar{L}_{B 2}+\bar{L}_{A 1} \bar{L}_{B 1}\right) \pm\left[\left(\bar{L}_{A 2}+\bar{L}_{B 2}+\bar{L}_{A 1} \bar{L}_{B 1}\right)^{2}-4 \bar{L}_{A 2} \bar{L}_{B 2}\right]^{1 / 2}\right\} .
$$

Assuming that the corresponding two eigenvectors of matrix $\overline{\mathbf{L}}$ are linearly independent, we obtain the explicit 
expression for the top surface morphological perturbation $\hat{h}_{k}^{*}\left(q^{*}\right)$ :

$$
\begin{aligned}
& \hat{h}_{k}^{*}=\bar{L}_{A 1} \frac{\lambda_{1}^{\frac{k-1}{2}}-\lambda_{2}^{\frac{k-1}{2}}}{\lambda_{1}-\lambda_{2}} \hat{h}_{2}^{*}+\bar{L}_{A 2}\left(-\bar{L}_{B 2} \frac{\lambda_{1}^{\frac{k-3}{2}}-\lambda_{2}^{\frac{k-3}{2}}}{\lambda_{1}-\lambda_{2}}+\frac{\lambda_{1}^{\frac{k-1}{2}}-\lambda_{2}^{\frac{k-1}{2}}}{\lambda_{1}-\lambda_{2}}\right) \hat{h}_{1}^{*} \quad(k \text { odd }), \\
& \hat{h}_{k}^{*}=\left(\frac{\lambda_{1}^{\frac{k}{2}}-\lambda_{2}^{\frac{k}{2}}}{\lambda_{1}-\lambda_{2}}-\bar{L}_{A 2} \frac{\lambda_{1}^{\frac{k-2}{2}}-\lambda_{2}^{\frac{k-2}{2}}}{\lambda_{1}-\lambda_{2}}\right) \hat{h}_{2}^{*}+\bar{L}_{A 2} \bar{L}_{B 1} \frac{\lambda_{1}^{\frac{k-2}{2}}-\lambda_{2}^{\frac{k-2}{2}}}{\lambda_{1}-\lambda_{2}} \hat{h}_{1}^{*} \quad(k \text { even }),
\end{aligned}
$$

which can be easily proved to be proportional to $\lambda^{k / 2}\left[\lambda=\max \left(\lambda_{1}, \lambda_{2}\right)\right]$ for real eigenvalues, or to $|\lambda|^{k / 2}(|\lambda|=$ $\left.\left|\lambda_{1}\right|=\left|\lambda_{2}\right|\right)$ for a complex eigenvalue pair, when the number of total layers $k \rightarrow \infty$. Thus, the effective stability of this multilayer system (defined for $k \gg 1$ ) is determined by the magnitude of eigenvalue $|\lambda|$. When the maximum $|\lambda|$ (with respect to wave number $q^{*}$ ) is larger than 1, the surface perturbation grows, leading to the instability of the multilayer film; otherwise, if $|\lambda| \leq 1$ for all the $q^{*}$ modes, the system is stable due to the decay of surface perturbations. According to Eqs. (34) and (36), the value of $|\lambda|$, and then the stability properties of the system, depend on 6 dimensionless parameters $\Gamma_{B}^{*} / v_{B}^{*}, \epsilon_{B}^{*}, v_{A}^{*}, l_{A}^{*}, l_{B}^{*}$, and $\gamma_{B}^{*}$ that are defined in Eq. (33).

The expression for $\lambda$ remains unchanged with respect to the interchange of $A$ and $B$ layers, since the eigenvalues of matrices $\overline{\mathbf{L}}=\overline{\mathbf{L}}_{B} \overline{\mathbf{L}}_{A}$ and $\overline{\mathbf{L}}^{\prime}=\overline{\mathbf{L}}_{A} \overline{\mathbf{L}}_{B}$, with $\overline{\mathbf{L}}_{A(B)}$ defined in Eq. (27), are the same. Thus, the stability properties of multilayer structure are determined by $|\lambda|$ and are identical in the $k \rightarrow \infty$ limit for the $A / B$ and $B / A$ stacking forms.

Note that the stability analysis presented here is for very large $k \gg 1$, while for the morphology of each layer $i(i=1, \ldots, k)$, or for the phase ${ }^{24}$ between two successive strained layers, which depends on the sign of $\hat{h}_{i}^{*} / \hat{h}_{i-1}^{*}$, the evolution Eqs. (26) and (28) [with the explicit expressions similar to Eq. (37)] should be used. Although the stability properties for $k \gg 1$ are independent of the type of the first layer or the interchange of $A$ and $B$ layers in the structure, the detailed morphology of each layer $i$ may be influenced by whether the first deposited layer is tensile or compressive, as seen from the expressions (26), (28), and (37).

\section{B. Stability results}

In this subsection, we present the results of stability diagrams for various (rescaled) material parameters and growth conditions. The stability properties are determined through the maximum magnitude of eigenvalue $\lambda$, with the use of the above analytic expressions and discussions. One of the factors that we focus on is the surface mobilities of strained layers. During the growth process, different mobilities of $A$ and $B$ layers results in different interface morphologies which are frozen in due to the deposition of upper layers, but which can influence the system's strain field and the evolution of top surface profile.

A common feature of the stability diagrams presented in this subsection is that the multilayer system can be effectively stabilized by large enough deposition rate $\left(v_{A}^{*}\right.$ and $\left.v_{B}^{*}\right)$, as also found in experiments. ${ }^{3,8}$ This is similar to single-layer film growth: ${ }^{20-22,27,28}$ for large enough deposition rate, the morphological perturbation of layer surface does not have enough time to develop through surface diffusion, since it is buried and frozen by fast subsequent materials deposition.

\section{Effect of different surface mobilities in strained layers}

Stability diagrams in the $l_{A}^{*}-v_{A}^{*}$ space are shown in Fig. 2 for different relative surface mobilities $\Gamma_{B}^{*}=\Gamma_{B} / \Gamma_{A}=$ $0.001,0.01,1$, and 100 (other parameters are $\epsilon_{B}^{*}=-1, l_{A}^{*}=l_{B}^{*}, v_{A}^{*}=v_{B}^{*}$, and $\gamma_{B}^{*}=1$ ). Important features are: larger deposition rate imply greater stability, very thick or very thin layers imply instability and for large $l_{A}$ and $l_{B}$, multilayer system loses coherency; larger relative mobility $\Gamma_{B}^{*}$ leads to more instability (note the larger unit-scale for $v_{A}^{*}$ in the inset).

To understand the effect due to different mobilities, we consider that the increase of $\Gamma_{B}^{*}=\Gamma_{B} / \Gamma_{A}$ corresponds to keeping the parameters of material $A$ (including $\Gamma_{A}, \epsilon_{A}$, and $\gamma_{A}$ ) unchanged, as seen in the rescaling Eq. (33), and to the increase of surface mobility $\Gamma_{B}$ of layer $B$. Consequently, for larger $\Gamma_{B}^{*}$, the morphological instability is enhanced due to a faster diffusion on surface of layer $B$ (driven by nonuniform strain energy distribution ${ }^{19,20}$ ), and then larger deposition rates $v_{A}^{*}$ and $v_{B}^{*}$ are needed to suppress it. In multilayer experiments, different mobilities result in different morphological profiles of $A$ and $B$ layers. ${ }^{4}$ Layers with high mobility exhibit the morphology of ripples with high peaks and deep valleys, while for layers with low mobility, flatter profile is observed. Therefore, increasing the surface mobility of either type of layer leads to rougher surface profile, which is more difficult to be smoothed out during subsequent growth. 
Our analytic expressions in last subsection can also explain this effect. Equations (36) and (34) show that the value of $|\lambda|$, which determines the effective stability of the system, depends on parameter $\Gamma_{B}^{*} / v_{B}^{*}$. Thus, when $\Gamma_{B}^{*}$ is larger, deposition rate $v_{B}^{*}$ should be correspondingly increased in order to keep the same value of $\Gamma_{B}^{*} / v_{B}^{*}$, or to retain the system within the region of stability.

\section{Interplay between mobility and global misfit of multilayer}

In the tensile/compressive multilayer structures, one important parameter is the global strain of the whole multilayer film with respect to substrate, which varies with the relative layer thickness $l_{B}^{*} / l_{A}^{*}\left(=l_{B} / l_{A}\right)$ and relative misfit $\epsilon_{B}^{*}$ $\left(=\epsilon_{B} / \epsilon_{A}\right)$. A qualitative measure of the global strain is provided by $l_{A} \epsilon_{A}+l_{B} \epsilon_{B}$. It is interesting to study the stability properties of systems with different global misfits, in particular the asymmetry for global tensile and compressive multilayers and its relationship to different surface mobilities of composite layers.

The effect of relative thickness $l_{B}^{*} / l_{A}^{*}$ (with fixed relative misfit $\epsilon_{B}^{*}=-1$ ) is depicted in Figs. 3 and 4 . In Fig. 3 (a) we plot the stability diagrams of $l_{A}^{*}$ versus $v_{A}^{*}$ for different values of $l_{B}^{*} / l_{A}^{*}$, with small relative mobility $\Gamma_{B}^{*}=0.01$. When $l_{B}^{*} / l_{A}^{*}$ increases from 1 , the stability boundary shifts to the lower value of $l_{A}^{*}$; but when $l_{B}^{*} / l_{A}^{*}<1$, the system is much more unstable, that is, very high deposition rate $v_{A}^{*}$ (orders of magnitude larger than the scale shown in the figure) is needed to stabilize the multilayer morphology.

Stability diagrams as a function of $l_{B}^{*} / l_{A}^{*}$ and $v_{A}^{*}$ (where the $A / B$ period thickness $l_{A}^{*}+l_{B}^{*}$ is held constant as in experiments) can illustrate this asymmetry more clearly, as shown in Fig. 4 (a) and (b). When $\Gamma_{B}^{*}<1$ ( $\left.>1\right)$, i.e., $\Gamma_{B}<(>) \Gamma_{A}$, the systems with $l_{B} / l_{A}>1(<1)$ are much more stable, corresponding to much smaller deposition rate $v_{A}^{*}\left(=v_{B}^{*}\right.$ here). When $\Gamma_{B}^{*}=1\left(\Gamma_{B}=\Gamma_{A}\right)$, Fig. $4(\mathrm{~b})$ shows that the stability diagram is much more symmetric. Also, for different relative mobilities $\Gamma_{B}^{*}$ [e.g., 0.01 and 0.001 as in Fig. 4 (a)], the system can display either a greater instability or a greater stability as the layer period thickness $l_{A}^{*}+l_{B}^{*}$ is increased. This can be understood through the stability diagram of $l_{A}^{*}$ versus $v_{A}^{*}$ in Fig. 2, where within different range of values of $l_{A}^{*}$, one can see that the stability can either increase or decrease with layer thickness $l_{A}^{*}$. This stability property with respect to period thickness is richer compared to the observation in InAs/AlAs experiments, where the modulation amplitude of the films was found to decrease with the decrease of period thickness. ${ }^{3}$

Figures 3 (b) and 5 exhibit the effect of relative misfit $\epsilon_{B}^{*}$, with fixed $l_{B}^{*} / l_{A}^{*}=1$. The asymmetric property, seen in Fig. 4, also appears in the stability diagrams of $l_{A}^{*}$ versus $v_{A}^{*}$ for different values of $\epsilon_{B}^{*}\left[\right.$ Fig. 3 (b)]. For $\Gamma_{B}^{*}=0.01$, when $\left|\epsilon_{B}^{*}\right|>1$ the instability is enhanced by the increase of the value of misfit, with the shrinking of stability region; however, when $\left|\epsilon_{B}^{*}\right|<1$ the $v_{A}^{*}$ values of stability boundary are much larger than the scale shown here, corresponding to a very unstable system. We can see the asymmetry more clearly in the $\epsilon_{B}^{*} v_{A}^{*}$ plot of Fig. 5 , where $l_{A}^{*}=l_{B}^{*}=0.5$ and when $\Gamma_{B}^{*}<1(>1)$, the systems with larger (smaller) misfit of layer $B$ [i.e., $\left|\epsilon_{B}^{*}\right|>1(<1)$ ] are much more stable.

Consequently, these stability diagrams in Figs. 3-5 show that when the sign of the multilayer global strain is the same as that of the layers with smaller surface atomic mobility, the system is more stable; otherwise, when the layers with larger mobility have relatively larger misfit strain or layer thickness, the modulations on the surface of these layers are enhanced due to faster surface diffusion and are hard to be suppressed by the subsequent deposition of layers with smaller mobility. Note that this result of stability asymmetry, which looks different for $\Gamma_{B}^{*}>1$ and $<1$, is in fact symmetric with respect to the exchange of $A$ and $B$ layers. E.g., when layer $A$ is compressive, layer $B$ is tensile, and $\Gamma_{B}^{*}=\Gamma_{B} / \Gamma_{A}<1$, we obtain from above analysis that global tensile multilayer film is more stable. If $A$ and $B$ are interchanged, layer $A^{\prime}$ (of material $B$ ) is tensile and layer $B^{\prime}$ (of material $A$ ) is compressive, with $\Gamma_{B^{\prime}}^{*}>1$; then according to above result, the global strain of the more stable system should be the sign of layer $A^{\prime}$, which is also tensile.

In recent experiments of InAs/AlAs short-period superlattices grown on $\operatorname{InP}(001){ }^{2-5}$ the system with large tensile global strain was observed to be stable. This is consistent with our theoretical results, since AlAs is tensile with respect to substrate InP, InAs is compressive, and importantly, Al has much smaller surface atomic mobility, leading to the possibility of stabilization for global tensile multilayer (corresponding to thicker AlAs layers) due to the above analysis. For the global compressive films (corresponding to thicker InAs layers), our calculations yield much larger instabilities, in agreement with the most recent experimental observation ${ }^{5}$ that although the multilayer structure under global compression exhibits weak lateral modulations as a whole, the system is in fact morphologically unstable due to a high degree of surface roughness and the reduced strength of lateral modulations can be attributed to irregular surface patterns.

To further compare with the experiments of InAs/AlAs, ${ }^{2-5}$ stability diagrams of $l_{B}^{*} / l_{A}^{*}$ versus $v_{A}^{*}$ (Fig. 4) should be used. Here layer $A$ corresponds to InAs and layer $B$ to AlAs. As shown in the figure, the stability boundary depends on the parameter values, in particular the relative mobility $\Gamma_{B}^{*}$ (which also depends on growth temperature as discussed below: when $\Gamma_{B}^{*}<1$ that applies here, higher temperature $T$ leads to larger $\left.\Gamma_{B}^{*}\right)$ and period thickness $l_{A}^{*}+l_{B}^{*}$. When $\Gamma_{B}^{*}$ is very small $\left(\right.$ e.g., $\left.=10^{-3}\right)$ and layer thickness $l_{A}^{*}+l_{B}^{*}$ is thin enough (e.g., $\left.=0.3\right)$, our results in 
Fig. 4 show that films with larger tensile global strain (corresponding to larger $l_{B}^{*} / l_{A}^{*}$ ) are more stable. However, when layer thickness $l_{A}^{*}+l_{B}^{*}$ increases (e.g., $=0.9$ ), we have opposite result for the multilayer under global tension: higher global strain leads to more instability. When relative mobility is larger (e.g., $\Gamma_{B}^{*}=10^{-2}$ ), different behavior can be obtained. Opposite to the case of $\Gamma_{B}^{*}=10^{-3}$, thinner $A / B$ layers $\left(l_{A}^{*}+l_{B}^{*}=0.3\right.$ in Fig. 4) result in more unstable global tensile film. Interestingly, for larger thickness $l_{A}^{*}+l_{B}^{*}=0.9$, from Fig. 4 we can get a maximum boundary value of $v_{A}^{*}$ (maximum instability) in the tensile region when $l_{B} / l_{A}$ is somewhat larger than 1 (i.e., the whole multilayer is somewhat tensile), which may correspond to the experimental observation of the maximum modulation for slightly global tensile InAs/AlAs film. ${ }^{2,4}$ Also there is a minimum boundary value $v_{A}^{*}$ for $l_{A}^{*}+l_{B}^{*}=0.9$, showing the maximum stability at some higher global strain.

Although our theoretical results qualitatively agrees with some experimental observations, for the quantitative comparison more realistic factors should be considered in our model. One important factor is the "wetting effect", ${ }^{29}$ especially for the multilayer structures like short-period superlattices where the thickness of each strained layer is very thin and then the film-substrate and layer-layer interface energies have to be carefully included in section II. This effect is absent in our calculation but applicable to the InAs/AlAs experimental system.

\section{Strain-balanced condition}

In some experiments for multilayer film growth, the strains caused by tensile and compressive layers are balanced. That is, the system fulfills the strain-balanced condition $l_{A} \epsilon_{A}+l_{B} \epsilon_{B}=0$, or equivalently, $l_{B}^{*} / l_{A}^{*}=-1 / \epsilon_{B}^{*}$, which makes the global misfit of multilayer film related to substrate equal to zero. One would expect that the corresponding stability diagrams are less asymmetric compared to the globally strained system studied above. This is seen in Fig. 6 for diagrams of $l_{B}^{*} / l_{A}^{*}$ versus $v_{A}^{*}$ (with fixed $l_{A}^{*}+l_{B}^{*}$ ). However, the asymmetry is still obvious, and from these stability diagrams of zero global strain we obtain an interesting property that for all values of relative mobilities $\Gamma_{B}^{*}=\Gamma_{B} / \Gamma_{A}$, the multilayer structures are more stable, that is, can be stabilized by lower rescaled deposition rates $v_{A}^{*}$ and $v_{B}^{*}$, when the relative misfit $\left|\epsilon_{B}^{*}\right|=\left|\epsilon_{B}\right| /\left|\epsilon_{A}\right|<1$ (or equivalently, $l_{B} / l_{A}>1$ ). Figure 6 should be compared to Fig. 5 (c) and $(\mathrm{d})$, since the later does not have a strain-balanced condition.

Note that this result is obtained for fixed material parameters $\Gamma_{A}, \epsilon_{A}$ and $\gamma_{A}$ (especially for rescaled deposition rate $v_{A(B)}^{*}=v_{A(B)} / v_{A}^{0}=v_{A(B)} \gamma_{A}^{2} /\left(\Gamma_{A} E^{\prime 3} \epsilon_{A}^{6}\right)$ that determines the degree of stability here), and one can obtain the same result with respect to fixed $B$ parameters by interchanging $A$ and $B$ in the calculation (see also similar diagrams for $\Gamma_{B}^{*}<1$ and $>1$ ). Thus, when we already have one layer material 1 (with misfit $\epsilon_{1}$ ), to make the multilayer system more stable, the other layer material 2 should be selected to have smaller misfit in relation to the substrate (i.e., $\left.\left|\epsilon_{2}\right|<\left|\epsilon_{1}\right|\right)$, which implies a larger layer thickness $l_{2}>l_{1}$ due to the strain-balanced condition.

Consequently, with the condition of strain balance, the multilayer structure with asymmetric $A$ and $B$ layers (consisting of alternately thin strained layers and thick layers with less misfit of opposite sign), are more stable compared to that with symmetric properties $\epsilon_{A}=-\epsilon_{B}$ and $l_{A}=l_{B}$, and more the asymmetry, more the stability. Also, this result is independent of the relative surface mobility of two types of layers. This theoretical prediction, which is different from the case of nonzero global strain studied above, should be related to different multilayer structures with different types of composite layers, and is yet to be verified by experiments.

In fact, this result can lead to the well-known fact of the heteroepitaxial growth: the system is more stable for less strained composite layers. When we fix material $A$ and select material $B$, the system is more stable for the material with smaller misfit $\epsilon_{B}$ (and then larger thickness $l_{B}$ ) according to our result; then, if we fix material $B$ and select other type of layer $A$, the material with smaller $\epsilon_{A}$ corresponds to more stable system. The repeat of this selection procedure leads to an obvious result that the most stable multilayer system is the structure in which both misfits $\epsilon_{A}$ and $\epsilon_{B}$ are as small as possible.

\section{Role of surface tension and growth temperature}

Our results for stability properties are not very sensitively dependent on relative surface tension $\gamma_{B}^{*}=\gamma_{B} / \gamma_{A}($ One of the major reasons may be that wetting effect ${ }^{29}$ is not considered here). See diagrams of $l_{A}^{*}$ versus $v_{A}^{*}$ in Fig. 7 (a), with $\Gamma_{B}^{*}=0.01$ and different $\gamma_{B}^{*}=0.1,1$, and 10. For relative tension $\gamma_{B}^{*}<1$, the stable region is smaller; but the stability diagrams do not change much if we compare the result of $\gamma_{B}^{*}=10$ to that of 0.1 (with the difference of two orders of magnitude for $\gamma_{B}^{*}$ ).

We also calculate the diagrams as a function of $l_{B}^{*} / l_{A}^{*}$ and $v_{A}^{*}$ in the strain-balanced condition, for different relative surface tension $\gamma_{B}^{*}$, as shown in Fig. 7 (b). The results depend on the relative mobility $\Gamma_{B}^{*}$ and period thickness $l_{A}^{*}+l_{B}^{*}$. For $l_{A}^{*}+l_{B}^{*}=0.9$ and $\Gamma_{B}^{*}=1$, the inset of Fig. 7 (b) shows that the stability of the system increases with the 
increase of $\gamma_{B}^{*}$, while for smaller $\Gamma_{B}^{*}=0.01$, the results are more complicated: for large $l_{B} / l_{A}, \gamma_{B}^{*}=1$ (i.e., $\gamma_{A}=\gamma_{B}$ ) corresponds to the most stable case, but for small $l_{B} / l_{A}$, larger $\gamma_{B}^{*}$ favours the stability.

Growth temperature is an important factor in real experiments. Our results in Sec. IV A show that the stability properties are affected by growth temperature $T$ via mobilities $\Gamma_{A}$ and $\Gamma_{B}$, since the rescaled parameter $\Gamma_{B}^{*}$ changes exponentially with $T: \Gamma_{B}^{*} \propto \exp \left[-\left(E_{B}-E_{A}\right) / k_{B} T\right]$ with $E_{A}$ and $E_{B}$ the activation energies of $A$ and $B$ materials. If $E_{B} \geq E_{A}$, as temperature $T$ increases, value of $\Gamma_{B}^{*}$ increases or remains unchanged; while for $E_{B}<E_{A}, \Gamma_{B}^{*}$ decreases with the increment of $T$. Also, the dimensionless deposition rates $v_{A}^{*}$ and $v_{B}^{*}$ decrease with temperature due to $v_{A(B)}^{*}=v_{A(B)} \gamma_{A}^{2} /\left(\Gamma_{A} E^{\prime 3} \epsilon_{A}^{6}\right)$ [see Eqs. (32) and (33)], $\Gamma_{A} \propto T^{-1} \exp \left(-E_{A} / k_{B} T\right)$, and then $v_{A(B)}^{*} \propto T \exp \left(E_{A} / k_{B} T\right)$. Thus, when temperature $T$ increases, for the case of $E_{B} \geq E_{A}$ (i.e., $\Gamma_{B}^{*} \leq 1$ ) larger (or fixed) value of $\Gamma_{B}^{*}$ and smaller deposition rate $v_{A(B)}^{*}$ render the system more unstable, according to the stability diagrams in e.g., Fig. 2. Similar result can be obtained for $E_{B}<E_{A}$ (i.e., $\Gamma_{B}^{*}>1$ ). As discussed above, when $T$ increases, value of $v_{A(B)}^{*}$ gets smaller, leading to more unstable system, but $\Gamma_{B}^{*}$ also decreases, favoring the stability of the film. Note that due to $0<E_{A}-E_{B}<E_{A}$, the reduction of $\Gamma_{B}^{*}$ is much slower than that of $v_{A(B)}^{*}$ as $T$ is increased. Hence, we still have the result that higher growth temperature enhances the instability of this multilayer structure, as expected.

\section{Discussion}

For the alternating tensile/compressive multilayer, when a layer $i$, which is tensile (compressive) with respect to the substrate, is grown with the presence of undulating surface, local strain energy concentration is created at the surface troughs with more stress relaxation at the peaks. When the next compressive (tensile) layer $i+1$ containing larger (smaller) atoms is deposited on it, the difference of strain energy density along the underlying surface drives the deposited atoms to the trough regions. ${ }^{24}$ This corresponds to the coupling of strain fields for two successive upper layers, while in reality the strain coupling is more complicated due to the influence of lower buried layers, which has been shown to decay exponentially with the distance from the growing surface [see Eq. (20)]. Nevertheless, some of our results in Sec. IV B can be explained by this simple two-layer coupling picture. When the layer thickness is too small, that is, the deposited material is not enough to smooth out the underlying troughs, the subsequent deposited layer $i+2$ will deepen the corrugations since it is of the same material as layer $i$. Thus, the surface perturbation is amplified by subsequent depositions of different layers, rendering the system unstable. On the other hand, when the layer thickness is too large, we have inverse morphology, that is, peak (valley) forms and continues to grow above the valley (peak) of underlying layer $i$, and then the surface morphological modulations can not decay with the layer stacking. Thus, for intermediate range of layer thickness, the multilayer is more stable, i.e., lower deposition rate is needed to reduce and suppress the surface undulations during each layer growth. Here, surface mobility plays an important role. Smaller mobility leads to slower stress-driven surface diffusion process, and then the surface morphology has less dependence on the corrugations of underlying layer. Consequently, the stability results in Fig. 2 can be reproduced, that is, the system is more unstable for too small or too large a layer thickness, higher relative mobility, and smaller deposition rate.

More interesting results are found for the multilayer films with global misfit with respect to the substrate. When the layers with smaller surface mobility have larger thickness or larger magnitude of misfit, that is, the sign of the multilayer global strain is the same as that of this type of layers, the system is more stable. This can also be seen in the above approximate picture of two-layer coupling. For example, for the growth of layer with small mobility, the surface evolution does not depend much on the undulations of the layer underneath, and then with large thickness the surface profile is flatter than that of underlying layer (as observed in experiments); when the next layer with larger mobility is deposited, the surface corrugation develops but is limited due to the smaller layer thickness. Therefore, the modulations are easier to suppress during the layer stacking procedure. This asymmetric property is consistent with the recent experimental results of $\mathrm{InAs} / \mathrm{AlAs}$ on $\mathrm{InP}(001)$, where the films under high global tension have been found to be stable ${ }^{2-5}$ while those under global compression are much more unstable. ${ }^{5}$ Note that the formation of lateral modulations observed in the whole multilayer film is due to the regular surface modulated pattern; for irregular surface pattern weak lateral modulations are observed, but the surface could be very rough, corresponding to unstable system. ${ }^{5}$

The other property we obtain for the tensile/compressive multilayer is related to the films with strain-balanced condition, i.e., zero global strain. We find that with this constraint, the multilayer structure is more stable if one type of strained layers is thicker and then has less misfit of opposite sign compared to the other type. To see this in experiments, one should compare multilayer films composed of different materials (such as $A / B$ and $A / B^{\prime}$ structures both with zero global strain, but $l_{B} \neq l_{B}^{\prime}$ and $\epsilon_{B} \neq \epsilon_{B^{\prime}}$ ). As discussed in Sec. IV B 3, this asymmetry property in fact leads to the well-known result that smaller misfit of each composite layer results in more stable system. 


\section{STRAINED/SPACER MULTILAYER STRUCTURES}

The other kind of multilayer that we are interested in is the structure with strained/spacer stacked layers. In this system, the strained layers (either tensile or compressive with respect to the substrate) and spacer layers are alternately deposited on a substrate of material $B$, such as $\operatorname{InAs} / \mathrm{GaAs}(001),{ }^{11-13} \mathrm{Ge} / \mathrm{Si}(001),{ }^{14-16}$ and $\mathrm{SiGe} / \mathrm{Si}(001) \cdot{ }^{9,10,18}$ The strained layers are odd-numbered $(i=1,3, \ldots)$, with nonzero misfit strains $\epsilon_{i} \neq 0$, while the even-numbered spacer layers $(j=2,4, \ldots)$ are of substrate material $B$, with zero misfit $\epsilon_{j}=\epsilon_{B}=0$. Here, we consider the case in which all the spacer layers are thick enough, such that the interface between a spacer layer $j$ and a strained layer $(j+1)$ is atomically flat $\left(\hat{h}_{j}=0\right.$ for even $j$ ) since the growth front of previously deposited strained layer is smoothed out. [If in the solution (22), one sets $\epsilon_{k}=\epsilon_{B}=0$, then $\sigma_{k}$ is negative and for thick enough spacer layers, Eq. (22) leads to a flat top surface of the spacer layer. This is also seen in many experiments.]

The experimental interest for this multilayer structure is different from that of tensile/compressive multilayer due to the presence of spacer layers. This system is morphologically unstable (as verified below), and 3D islands develop for each strained layer with the self-organized formation of multisheet arrays of islands along the growth direction. It has been observed that both size and regularity of islands increase with the increment of deposition layers, and then saturate for large enough layer numbers. In the linear analysis presented here, this property of the island size can be explained through the study of kinetic critical thickness for the onset of 3D instability, with details shown in Sec. VB.

\section{A. Elastic analysis and morphological evolution}

The recursive procedure for elastic analysis presented in Sec. II can be applied to the strained/spacer multilayer structures. The corresponding results are simpler due to the zero misfit of spacer layers and the flat strained/spacer interfaces (i.e., $\epsilon_{j}=0, \hat{h}_{j}=0$ for even $j$ ), and are different for the odd-numbered (strained) and even-numbered (spacer) layers. Consider a $k$-layer system with top layer $k$ strained with respect to the substrate (i.e., with odd number $k$ ). Thus, the interfaces between odd- and even-numbered layers (that is, $i /(i-1)$ interfaces with odd $i$ and $3 \leq i \leq k)$ in Fig. 1 (a) are planar. The effective boundary conditions at the top surface $z=\bar{h}_{k}$ and the interfaces $z=\bar{h}_{i}(0 \leq i \leq k-2)$ below layer $(k-1)$ are the same as Eqs. (16) and (18), while at planar $k /(k-1)$ interface the special boundary condition (17) should be revised as $(\alpha=x, y, z)$

$$
\left.\hat{\sigma}_{\alpha z, k}\right|_{k}=\left.\hat{\sigma}_{\alpha z, k-1}^{n}\right|_{k} \quad \text { and }\left.\quad \hat{u}_{\alpha, k}\right|_{k}=\left.\hat{u}_{\alpha, k-1}\right|_{k-1}+\left.\hat{u}_{\alpha, k-1}^{n}\right|_{k} .
$$

Similar to the discussion in Sec. II, we obtain an elastically effective system composed of an undulating top free surface and a planar interface between top single layer $k$ and an effective semi-infinite elastic medium of field $\left.u_{\alpha, k-1}^{n}\right|_{k}$ with boundary condition (38), to first order of perturbations and with the assumption of identical elastic constants. To determine the elastic fields recursively, we need to consider the system with smaller number of layers. For a $m$-layer system with $1<m<k$, the above results can be directly applied with the replacement of $k$ by $m$ if layer number $m$ is odd. However, for even number $m$, the results are somewhat different due to the flat top surface of even-numbered spacer layer $m$. The corresponding effective system is in fact composed of top layer $m$, with a planar free surface, and underlying effective semi-infinite medium of elastic field $\left.u_{\alpha, m-1}^{n}\right|_{m}$, with a nonplanar buried interface. The boundary conditions are different from those of Eqs. (16) and (38): At planar top free surface $z=\bar{h}_{m}$,

$$
\left.\hat{\sigma}_{\alpha z, m}\right|_{m}=0,
$$

while at rough interface $z=\bar{h}_{m-1}$,

$$
\begin{gathered}
\left.\hat{\sigma}_{\alpha z, m}\right|_{m}=\left.\hat{\sigma}_{\alpha z, m-1}^{n}\right|_{m} \\
\left.\hat{u}_{\beta, m}\right|_{m}=\left.\hat{u}_{\beta, m-1}\right|_{m-1}+\left.\hat{u}_{\beta, m-1}^{n}\right|_{m} \text { and } \\
-\bar{u}_{m-1} \hat{h}_{m-1}+\left.\hat{u}_{z, m}\right|_{m}=\left.\hat{u}_{z, m-1}\right|_{m-1}+\left.\hat{u}_{z, m-1}^{n}\right|_{m} .
\end{gathered}
$$

(Here $\alpha=x, y, z$ and $\beta=x, y$.)

According to the procedure in sections II and III, we can determine the elastic energy density and then derive the evolution equation for surface morphological perturbation of top strained layer $k$ :

$$
\begin{aligned}
\partial \hat{h}_{k}(q, t) / \partial t & =\sigma_{k} \hat{h}_{k}(q, t)+E^{\prime} \Gamma_{k} \epsilon_{k} q^{3} e^{-q v_{k} t} \\
& \times\left[\sum_{j=1}^{\frac{k-1}{2} e^{-q} \sum_{i=2 j}^{k-1} l_{i}} \epsilon_{2 j-1} \hat{h}_{2 j-1}(q)-e^{-q \sum_{i=1}^{k-1} l_{i}} \epsilon_{1} \hat{\zeta}(q)\right]
\end{aligned}
$$


which in fact can be obtained from Eq. (20) of general case by considering $\epsilon_{j}=0$ and $\hat{h}_{j}=0$ for spacer layer $j$. For simplicity, here we assume the planar film-substrate interface, i.e., $\hat{\zeta}=0$, and the initial perturbation on the top surfaces of substrate and of all the spacer layers, from which the strained layers start growing, to be $\hat{h}^{0}(q)$, which can arise from the background roughness due to the noise of deposition flux even if the starting surface is perfectly flat. Then the solution of evolution equation (41) is expressed as

$$
\hat{h}_{k}(q, t)=A_{k}(q, t) \hat{h}^{0}(q),
$$

where $A_{k}(q, t)$ is the morphological perturbation amplitude for top surface of strained layer $k$. For $k=1, A_{1}(q, t)=$ $\exp \left(\sigma_{1} t\right)$ according to Eq. (A.1) and the result of single-layer film growth; for $k=3$, we have

$$
A_{3}(q, t)=e^{\sigma_{3} t}+\frac{\Gamma_{3} E^{\prime} \epsilon_{1} \epsilon_{3} q^{3}}{q v_{3}+\sigma_{3}} e^{\sigma_{1} l_{1} / v_{1}-q l_{2}}\left(e^{\sigma_{3} t}-e^{-q v_{3} t}\right) ;
$$

and for $k \geq 5$,

$$
\begin{aligned}
A_{k}(q, t) & =L_{k, 1}(q, t)+L_{k, 2}(q, t)\left\{\bar{L}_{k-2,1}(q)\right. \\
& \left.+\sum_{i=2}^{\frac{k-5}{2}}\left[\prod_{j=i+1}^{\frac{k-3}{2}} \bar{L}_{2 j+1,2}(q)\right] \bar{L}_{2 i+1,1}(q)+\left[\prod_{j=2}^{\frac{k-3}{2}} \bar{L}_{2 j+1,2}(q)\right] \bar{A}_{3}(q)\right\},
\end{aligned}
$$

where

$$
\begin{aligned}
L_{k, 1}(q, t) & =e^{\sigma_{k} t}-\frac{\Gamma_{k} \epsilon_{k}}{\Gamma_{k-2} \epsilon_{k-2}} \frac{q v_{k-2}+\sigma_{k-2}}{q v_{k}+\sigma_{k}} \frac{e^{\sigma_{k} t}-e^{-q v_{k} t}}{e^{\sigma_{k-2} l_{k-2} / v_{k-2}}-e^{-q l_{k-2}}} e^{\sigma_{k-2} l_{k-2} / v_{k-2}-q\left(l_{k-1}+l_{k-2}\right)}, \\
L_{k, 2}(q, t) & =\frac{\Gamma_{k} \epsilon_{k}}{\Gamma_{k-2} \epsilon_{k-2}} \frac{e^{-q l_{k-1}}}{q v_{k}+\sigma_{k}} \frac{e^{\sigma_{k} t}-e^{-q v_{k} t}}{e^{\sigma_{k-2} l_{k-2} / v_{k-2}-e^{-q l_{k-2}}}} \\
& \times\left[\left(q v_{k-2}-\Gamma_{k-2} \gamma_{k-2} q^{4}\right) e^{-q l_{k-2}}+\Gamma_{k-2} E^{\prime} \epsilon_{k-2}^{2} q^{3} e^{\sigma_{k-2} l_{k-2} / v_{k-2}}\right],
\end{aligned}
$$

and $\bar{A}_{3}(q), \bar{L}_{2 j+1,1}(q)$, and $\bar{L}_{2 j+1,2}(q)[2 \leq j \leq(k-3) / 2]$ are obtained by replacing $k$ with $2 j+1$ and $t$ with $l_{2 j+1} / v_{2 j+1}$ in $A_{3}(q, t), L_{k, 1}(q, t)$, and $L_{k, 2}(q, t)$, respectively. Formula (45) shows that when the underlying spacer layer is very thick, i.e., $l_{k-1} \gg 1$, we have $L_{k, 1}(q, t) \rightarrow \exp \left(\sigma_{k} t\right)$ and $L_{k, 2}(q, t) \rightarrow 0$. Then the single-layer solution similar to Eq. (A.1): $\hat{h}_{k}(q, t)=\exp \left(\sigma_{k} t\right) \hat{h}^{0}(q)$ is recovered for this multilayer structure with any value of the layer number $k$, as expected.

Consequently, we have derived the explicit solution [Eqs. (42)-(45)] for the surface morphological perturbation, in terms of material and growth parameters for all the deposited layers $(1 \leq i \leq k): \Gamma_{i}, \epsilon_{i}, \gamma_{i}, l_{i}, v_{i}$, and the elastic constant $E^{\prime}$. Also, the above expressions can be directly used to determine the early evolution and stability properties of stacked strained/spacer structures, for which the composite materials and growth conditions of different layers could be different (non-periodic structure) or the same (periodic structure).

\section{B. Results: kinetic critical thickness}

Here we apply the general results derived above to periodic strained/spacer structure studied in many experiments, ${ }^{9-16,18}$ for which, all the strained layers (odd-numbered) consist of the same material $A$, with the same deposition rate $v_{A}$ and average thickness $l_{A}$, and the thickness of each spacer layer (even-numbered) is fixed as $l_{B}$. Thus, from Eq. (44) the perturbation amplitude $A_{k}$ (for odd $k$ and $k \geq 5$ ) is simplified as

$$
A_{k}(q, t)=L_{1}(q, t)+L_{2}(q, t)\left[\bar{L}_{1}(q) \frac{1-\bar{L}_{2}(q)^{\frac{k-5}{2}}}{1-\bar{L}_{2}(q)}+\bar{L}_{2}(q)^{\frac{k-5}{2}} \bar{A}_{3}(q)\right],
$$

where $L_{1(2)}(q, t)$ corresponds to $L_{k, 1(2)}(q, t)$ in formula (45) in which one has set $\sigma_{i}=\sigma_{A}, \Gamma_{i}=\Gamma_{A}, \epsilon_{i}=\epsilon_{A}, \gamma_{i}=\gamma_{A}$, $l_{i}=l_{A}$, and $v_{i}=v_{A}(i=k, k-2)$, and $\bar{L}_{1(2)}(q)=L_{1(2)}\left(q, t=l_{A} / v_{A}\right)$, so that all of them are independent of layer number. For smaller $k$, the amplitudes are $A_{1}(q, t)=\exp \left(\sigma_{A} t\right)$ for single-layer film, and

$$
A_{3}(q, t)=e^{\sigma_{A} t}+\frac{\Gamma_{A} E^{\prime} \epsilon_{A}^{2} q^{3}}{q v_{A}+\sigma_{A}} e^{\frac{\sigma_{A} l_{A}}{v_{A}}-q l_{B}}\left(e^{\sigma_{A} t}-e^{-q v_{A} t}\right)
$$


as obtained directly from Eq. (43).

¿From Eqs. (46) and (47) we find that when the growth time is large enough, i.e., $t \gg 1$, the perturbation amplitude $A_{k}(q, t)$ is proportional to $\exp \left(\sigma_{A} t\right)$, with positive perturbation growth rate for small wavenumber $q$ as in the case of single-layer film. In Fig. 8, we show the maximum value of perturbation amplitude (with respect to wavenumber q) $A_{k}^{\max }(t)$ as a function of growth time $t$ for different layer numbers, and note the positive growth rate. Thus, similar to the tensile/compressive structure discussed in Sec. IV, essentially this strained/spacer multilayer system is morphologically unstable. However, different from the tensile/compressive films, the system here cannot be effectively stabilized (with the decay of initial morphological perturbation) in certain range of growth parameters. This is due to the fact that the surface perturbation amplitude always increases with layer number $k$, i.e., $A_{k}(q, t)>A_{k-2}(q, t)>$ $\ldots>A_{3}(q, t)>A_{1}(q, t)>0$ for certain wavenumber $q$ and growth time $t\left(=l_{A} / v_{A}\right)$, which can be proved analytically from Eqs. (46) and (47) (see also numerical result of maximum perturbation amplitude shown in Fig. 8), and can be attributed to the coupling of interface perturbations of all the buried layers exhibited in Eq. (41). Also, if the spacer layer thickness $l_{B}$ is large enough that $\bar{L}_{2}(q)<1$ for any mode $q$, we find the asymptotic expression for amplitude $A_{k}$, as $k \rightarrow \infty$, as

$$
A_{k}(q, t) \rightarrow L_{1}(q, t)+L_{2}(q, t) \bar{L}_{1}(q) /\left[1-\bar{L}_{2}(q)\right] .
$$

Nevertheless, during the growth process the surface instability of top strained layer can be kinetically stabilized up to a critical thickness, as in the single-layer film growth. ${ }^{20}$ For growing layer below this kinetic critical thickness, that is, at early time of layer deposition, the surface perturbation does not develop fast enough compared to the deposition of materials flux, and then is not observable. The perturbation becomes apparent relative to the background disturbance only when the layer is sufficiently thick and then the initial undulation $\hat{h}^{0}$ for the growth of top strained layer is substantially amplified. As in the study of single-layer film, ${ }^{22}$ we use $\ln \left(\hat{h} / \hat{h}^{0}\right) \sim 1$ as the amplification limit, or equivalently, we use

$$
\ln \left[A_{k}^{\max }\left(t=t_{c}\right)\right]=1
$$

with $t_{c}=\left.h_{c}\right|_{k} / v_{A}$, as a measure of the kinetic critical thickness $\left.h_{c}\right|_{k}$ for a $k$-layer system (see Fig. 8). In Eq. (49), we have assumed that the surface morphological property is dominated by the mode of maximum perturbation amplitude $\left(A_{k}^{\max }\right)$, that is, by the wavelength of the strongest instability which, in single-layer case, may correspond to the wavelength of the surface roughness pattern observed in real experiments. ${ }^{30,31}$

The rescaled kinetic critical thickness $\left.h_{c}\right|_{k} /\left.h_{c}\right|_{k=1}$ (with $\left.h_{c}\right|_{k=1}$ the result for single-layer growth) is plotted as a function of layer number $k$ in Fig. 9, according to Eqs. (46) and (49). We choose the material parameters of Ge/Si(001) multilayer growth, ${ }^{15,16}$ with strained layer thickness $l_{A}=4 \mathrm{ML}$, deposition rate $v_{A}=1 \mathrm{ML} / \mathrm{min}$, temperature $T=550$ ${ }^{\circ} \mathrm{C}$, and different spacer layer thickness $l_{B}=8 \mathrm{~nm}, 8.5 \mathrm{~nm}$, and $9.5 \mathrm{~nm}$. (Typical Ge layers are rather thin and usually expressed in units of monolayers, while the Si spacer layers are relatively thick and appropriately expressed in nanometers, as in experiments. ${ }^{14-16}$ We use the conversion $1 \mathrm{ML}=0.1457 \mathrm{~nm}$ for Ge monolayers ${ }^{15}$ in our calculations.) For surface mobility $\Gamma_{k}$, the definitive measurement of its absolute value over a range of temperature is lacking, and we determine it from the expression of single-layer result: $\left.h_{c}\right|_{k=1}=256 v_{A} \gamma_{A}^{3} /\left(27 \Gamma_{A} \epsilon_{A}^{8} E^{\prime 4}\right)$, as well as the observed value of $\left.h_{c}\right|_{k=1}$, corresponding to the experimentally measured 2D to 3D transition thickness, in Ge/Si(001) growth. As illustrated in Fig. 9, the kinetic critical thickness $\left.h_{c}\right|_{k}$ first decreases monotonically and rapidly as the number $k$ of deposited layers increases, and then slowly until it converges to a saturation value at large enough number $k$. This convergence effect is due to the asymptotic result of perturbation amplitude $A_{k}$ [see Eq. (48)] when the spacer layer is sufficiently thick and then $\bar{L}_{2}(q)<1$. Also, from the comparison of different symbols (corresponding to different spacer layer thicknesses $l_{B}$ ) in Fig. 9, we find that $\left.h_{c}\right|_{k}$ is reduced more rapidly for thinner spacer layer, approaching a lower saturation value at larger number $k$. This can be attributed to the stronger coupling between top surface and the underlying layers and interfaces for smaller $l_{B}$, as seen in Eq. (41).

These results shown in Fig. 9, both for the behavior of $\left.h_{c}\right|_{k}$ versus $k$ and for the effect of spacer thickness $l_{B}$, are in good agreement with the recent experimental observations ${ }^{13-16}$ of critical thickness for $2 \mathrm{D}-3 \mathrm{D}$ growth transition. The properties of kinetic critical thickness presented here can explain some experimental results for islands (quantum dots) growth. When the thickness of growing film exceeds $\left.h_{c}\right|_{k}$, the morphological instability occurs, exhibiting as surface ripples or corrugations which have been observed as the precursor to the coherent islands formation. ${ }^{30,31}$ Thus, smaller $\left.h_{c}\right|_{k}$ not only corresponds to the earlier occurrence of islands during growth, but also results in larger island size since it implies more time for the development of 3D islands if the nominal thickness of top strained layer is fixed. Consequently, the results in Fig. 9 correspond to the experimental phenomenon of the increase and then saturation of islands size in upper strained layers. ${ }^{13,15,16}$

To further study the effect of layer thickness, we plot the rescaled critical thickness $\left.h_{c}\right|_{k} /\left.h_{c}\right|_{k=1}$ as a function of spacer layer thickness $l_{B}$ in the inset of Fig. 9, for different layer numbers $k=3,5,7$, and 11 . $\left.h_{c}\right|_{k}$ increases as the spacer thickness $l_{B}$ increases and the layers number $k$ decreases, in agreement with Fig. 9 and recent Ge/Si(001) 
experiments. ${ }^{16}$ When $l_{B}$ is large enough, the increase of $\left.h_{c}\right|_{k}$ becomes much slower, with the value approaching that of the single layer growth, as analyzed in Sec. VA. The relationship between the rescaled $\left.h_{c}\right|_{k}$ and the thickness $l_{A}$ of buried strained layers is presented in Fig. 10, which shows the opposite effect of $l_{A}$. Larger $l_{A}$ causes rougher morphology of buried interfaces, and hence the enhanced perturbation on surface as a result of the strain fields coupling [see also Eq. (41)]. Consequently, for larger $l_{A}$ instability occurs earlier, with smaller value of $\left.h_{c}\right|_{k}$.

The behavior of kinetic critical thickness $\left.h_{c}\right|_{k}$ with respect to misfit strain $\epsilon_{A}$ and deposition rate $v_{A}$ is shown in Fig. 11 (a) and (b). The results of $k=1$ there correspond to those of single-layer growing film with single component, for which the kinetic critical thickness is found to follow the $\epsilon_{A}^{-8}$ power law and increase linearly with $v_{A} \cdot{ }^{20}$ Similar properties can also be obtained in our calculations for strained/spacer multilayers with layer number $k>1$ and at small misfit [see the range $\epsilon_{A}<4 \%$ in Fig. 11 (a)] and large deposition rate [see Fig. 11 (b) and its inset]. However, small deviations do occur for large $\epsilon_{A}$ and very small $v_{A}$, as illustrated in Fig. 11, especially for large $k$ systems. Around and beyond the misfit of 4\%, Fig. 11 (a) shows that $\left.h_{c}\right|_{k}$ for $k>1$ decreases faster than $\epsilon_{A}^{-8}$ as misfit increases, with the behavior deviated from the power law; also, as presented in Fig. 11 (b), the linear relationship between $\left.h_{c}\right|_{k}$ and $v_{A}$ is deviated slightly at very small deposition rate. These deviations are more obvious for larger number $k$. But when $k$ is large enough ( $k \geq 11$ for the parameters of Fig. 11), the graphs of $\left.h_{c}\right|_{k}$ for different $k$ are almost identical in Fig. 11 (a) and (b), due to the convergence effect obtained analytically in Eq. (48) and shown numerically in Fig. 9 for large $k$.

Note that the kinetic critical thickness $\left.h_{c}\right|_{k}$ studied here, which is for the onset of morphological instability during layer growth, is different from the wetting layer thickness $h_{\mathrm{WL}}$ which corresponds to equilibrium critical thickness ${ }^{32}$ (postgrown and thermodynamically stable) for the 3D island formation. Usually $\left.h_{c}\right|_{k}>h_{\mathrm{WL}}$ due to the consumption of wetting layer in the formation of 3D islands. E.g., in the molecular-beam epitaxy (MBE) experiment of single layer Ge on $\operatorname{Si}(100),\left.{ }^{32} h_{c}\right|_{k=1}=3.7 \mathrm{ML}$ at temperature $T=700{ }^{\circ} \mathrm{C}$, while $h_{\mathrm{WL}}=3 \mathrm{ML}$.

For this stacked strained/spacer multilayer, due to the presence of spacer layers which are of the substrate material, the mechanism for morphological instability is more analogous to that of single-layer film growth compared to the tensile/compressive structure. But the crucial factor is still the coupling of strain fields in different strained layers. Also, from the general expressions in Sec. III, it is straightforward to obtain the results of morphological perturbation for the case of nonplanar and rough spacer layer $B$, that is, $\epsilon_{i}=0$ but $\hat{h}_{i} \neq 0$ for even number $i$. The corresponding stability properties and behavior of kinetic critical thickness are more complicated than those of the system with planar spacer layers studied above, due to the rough strained/spacer interfaces leading to a stronger coupling of strain fields.

\section{CONCLUSIONS}

We have studied the early morphological evolution and stability/instability properties of coherent multilayer structures with the stacking of layers of different materials. Generally, the diffusion in the bulk film is assumed to be negligible, thus the phenomena observed in the whole multilayer films, such as the lateral composition modulations in tensile/compressive multilayer and the vertically correlated arrays of islands in stained/spacer system, are in fact surface-induced and arise through the stacking of layers with the coupling of strain fields between surface and underlying layers. We have proposed a recursive procedure to directly calculate the elastic fields of a multilayer system to first order of perturbations. Then, based on the surface diffusion processes, the evolution equation of surface morphological perturbation is expressed through the elastic and morphological properties of all the layers underneath, including the misfit strains and buried interface perturbations. From the exact solution of this first-order equation, which is in terms of material parameters and growth conditions for all the deposited layers, we have determined the properties of stress-driven morphological instability for both the tensile/compressive and strained/spacer multilayers.

In this paper, we have only applied our results of early morphological evolution, derived in sections II and III, to two kinds of periodic multilayer structures, but more complicated structures can be studied similarly. Note that for multilayers with very thin layer thickness, i.e., for short-period superlattices, although our results can qualitatively explain and predict some experimental observations, more realistic factors such as the wetting effect should be included in our model to quantitatively describe the system properties. Also, studies beyond the linear analysis are needed to determine the details of morphological modulations or islands evolution during the multilayer growth. All these theoretical results can be useful in the fabrication of high-quality and novel multilayer structures.

\section{Acknowledgments}

This work was supported by the NSERC of Canada. 


\section{APPENDIX: RESULTS FOR FIRST AND SECOND LAYER PERTURBATIONS}

The morphological perturbation $\hat{h}_{1}$ for first deposited layer 1 is the same as that derived in the case of single-layer film growth. For the planar film-substrate interface $(\zeta=0)$, it is

$$
\hat{h}_{1}(q, t)=e^{\sigma_{1} t} \hat{h}^{0}(q)
$$

with the assumption of identical elastic constants. ${ }^{19}$ Here $\sigma_{1}=\Gamma_{1}\left(E^{\prime} \epsilon_{1}^{2} q^{3}-\gamma_{1} q^{4}\right)$ and $\hat{h}^{0}(q)$ is the initial perturbation. For the layer with fixed thickness $l_{1}$, Eq. (A.1) leads to

$$
\hat{h}_{1}(q)=e^{\sigma_{1} l_{1} / v_{1}} \hat{h}^{0}(q) .
$$

The perturbation $\hat{h}_{2}$ for second deposited layer can be calculated from the method in Sec. II and the expression (A.2) for $\hat{h}_{1}$, with the results

$$
\begin{aligned}
\hat{h}_{2}(q, t) & =\frac{1}{q v_{2}+\sigma_{2}}\left[\left(q v_{2}+\Gamma_{2}\left(E^{\prime} \epsilon_{2} \epsilon_{1} q^{3}-\gamma_{2} q^{4}\right)\right) e^{\sigma_{2} t}\right. \\
& \left.+\Gamma_{2} E^{\prime} \epsilon_{2}\left(\epsilon_{2}-\epsilon_{1}\right) q^{3} e^{-q v_{2} t}\right] e^{\sigma_{1} l_{1} / v_{1}} \hat{h}^{0}(q),
\end{aligned}
$$

where $\sigma_{2}=\Gamma_{2}\left(E^{\prime} \epsilon_{2}^{2} q^{3}-\gamma_{2} q^{4}\right)$, and

$$
\hat{h}_{2}(q)=\hat{h}_{2}\left(q, t=l_{2} / v_{2}\right)
$$

when second layer has fixed thickness $l_{2}$.

In some real growth systems, ${ }^{33,34}$ the substrate surface is nonplanar, corresponding to the case of rough $(\zeta \neq 0)$ film-substrate interface. The elastic state of both film and substrate is altered by this rough interface, with the detailed results shown in Ref. 28. Here we use these elastic results to obtain the expression of first layer perturbation for single-component film:

$$
\hat{h}_{1}(q, t)=\frac{1}{q v_{1}+\sigma_{1}}\left[\left(q v_{1}-\Gamma_{1} \gamma_{1} q^{4}\right) e^{\sigma_{1} t}+\Gamma_{1} E^{\prime} \epsilon_{1}^{2} q^{3} e^{-q v_{1} t}\right] \hat{\zeta}(q),
$$

where we have assumed the initial condition $\hat{h}_{1}(q, t=0)=\hat{\zeta}(q)$. Note that the contribution of nonzero interface morphology $\zeta$ is reflected in the second term of r.h.s. of Eq. (A.5), which is exponentially damped as the film grows. Therefore, the occurrence of instability is still determined by the real part of perturbation growth rate $\sigma_{1}$, as in the case of flat film-substrate interface [see Eq. (A.1)] ${ }^{19-22,27,28}$ and the introducing of nonzero $\zeta$ does not influence the stability/instability condition for single-layer film growth. On the other hand, Eq. (A.5) shows that compared to the case of flat interface $\zeta=0$, the kinetic critical thickness for the onset of 3D instability ${ }^{20}$ (see also Sec. V for the definition) decreases for rough interface $(\zeta \neq 0)$. Also, the nonzero substrate roughness $\zeta$ can affect the detailed film morphology, as found in experiments. ${ }^{33,34}$

The second layer surface perturbation can then be calculated for rough film-substrate interface, with the result:

$$
\begin{aligned}
\hat{h}_{2}(q, t) & =\frac{1}{q v_{2}+\sigma_{2}}\left\{\frac{1}{q v_{1}+\sigma_{1}}\left[\left(q v_{1}-\Gamma_{1} \gamma_{1} q^{4}\right) e^{\sigma_{1} l_{1} / v_{1}}+\Gamma_{1} E^{\prime} \epsilon_{1}^{2} q^{3} e^{-q l_{1}}\right]\right. \\
& \times\left[\left(q v_{2}+\Gamma_{2}\left(E^{\prime} \epsilon_{2} \epsilon_{1} q^{3}-\gamma_{2} q^{4}\right)\right) e^{\sigma_{2} t}+\Gamma_{2} E^{\prime} \epsilon_{2}\left(\epsilon_{2}-\epsilon_{1}\right) q^{3} e^{-q v_{2} t}\right] \\
& \left.+\Gamma_{2} E^{\prime} q^{3} \epsilon_{2} \epsilon_{1}\left(e^{-q v_{2} t}-e^{\sigma_{2} t}\right) e^{-q l_{1}}\right\} \hat{\zeta}(q) .
\end{aligned}
$$

Similar to Eqs. (A.2) and (A.4), the surface morphological perturbations of first and second layer with fixed layer thicknesses, i.e., $\hat{h}_{1}(q)$ and $\hat{h}_{2}(q)$, can be obtained by substituting $t=l_{1} / v_{1}$ and $t=l_{2} / v_{2}$ into Eqs. (A.5) and (A.6), respectively.

* Electronic address: huang@csit.fsu.edu; Present address: School of Computational Science and Information Technology, Florida State University, Tallahassee, Florida 32306-4120

$\dagger$ Electronic address: desai@physics.utoronto.ca 
1 V. A. Shchukin and D. Bimberg, Rev. Mod. Phys. 71, 1125 (1999).

2 A. G. Norman, S. P. Ahrenkiel, H. Moutinho, M. M. Al-Jassim, A. Mascarenhas, J. Mirecki Millunchick, S. R. Lee, R. D. Twesten, D. M. Follstaedt, J. L. Reno, and E. D. Jones, Appl. Phys. Lett. 73, 1844 (1998).

3 S. P. Ahrenkiel, A. G. Norman, M. M. Al-Jassim, A. Mascarenhas, J. Mirecki-Millunchick, R. D. Twesten, S. R. Lee, D. M. Follstaedt, and E. D. Jones, J. Appl. Phys. 84, 6088 (1998).

${ }^{4}$ R. D. Twesten, D. M. Follstaedt, S. R. Lee, E. D. Jones, J. L. Reno, J. Mirecki Millunchick, A. G. Norman, S. P. Ahrenkiel, and A. Mascarenhas, Phys. Rev. B 60, 13619 (1999).

5 A. G. Norman, H. R. Moutinho, M. M. Al-Jassim, A. Mascarenhas, S. R. Lee, J.Mirecki Millunchick, J. L. Reno, and D. M. Follstaedt, Appl. Phys. Lett. (to be published).

${ }^{6}$ A. Ponchet, A. Rocher, J.-Y. Emery, C. Starck, and L. Goldstein, J. Appl. Phys. 74, 3778 (1993).

7 A. Ponchet, A. Rocher, A. Ougazzaden, and A. Mircea, J. Appl. Phys. 75, 7881 (1994).

8 A. Ponchet, A. LeCorre, A. Godefroy, S. Salaün, and A. Poudoulec, J. Cryst. Growth 153, 71 (1995).

9 J. Tersoff, C. Teichert, and M. G. Lagally, Phys. Rev. Lett. 76, 1675 (1996).

10 C. Teichert, M. G. Lagally, L. J. Peticolas, J. C. Bean, and J. Tersoff, Phys. Rev. B 53, 16334 (1996).

11 Q. Xie, A. Madhukar, P. Chen, and N. P. Kobayashi, Phys. Rev. Lett. 75, 2542 (1995).

12 J. C. González, F. M. Matinaga, W. N. Rodrigues, M. V. B. Moreira, A. G. de Oliveira, M. I. N. da Silva, J. M. C. Vilela, M. S. Andrade, D. Ugarte, and P. C. Silva, Appl. Phys. Lett. 76, 3400 (2000).

13 Y. Nakata, Y. Sugiyama, T. Futatsugi, N. Yokoyama, J. Cryst. Growth 175/176, 713 (1997).

14 O. G. Schmidt, O. Kienzle, Y. Hao, K. Eberl, and F. Ernst, Appl. Phys. Lett. 74, 1272 (1999).

15 V. Le Thanh, V. Yam, P. Boucaud, F. Fortuna, C. Ulysse, D. Bouchier, L. Vervoort, and J. M. Lourtioz, Phys. Rev. B 60, 5851 (1999).

16 V. Le Thanh, V. Yam, P. Boucaud, Y. Zheng, and D. Bouchier, Thin Solid Films 369, 43 (2000).

17 F. Liu, S. E. Davenport, H. M. Evans and M. G. Lagally, Phys. Rev. Lett. 82, 2528 (1999).

18 H. Lafontaine, N. L. Rowell, and S. Janz, Appl. Phys. Lett. 72, 2430 (1998).

19 R. J. Asaro and W. A. Tiller, Metall. Trans. 3, 1789 (1972); M. A. Grinfeld, Sov. Phys. Dokl. 31, 831 (1987); D. J. Srolovitz, Acta Metall. 37, 621 (1989).

${ }^{20}$ B. J. Spencer, P. W. Voorhees, and S. H. Davis, Phys. Rev. Lett. 67, 3696 (1991); J. Appl. Phys. 73, 4955 (1993).

21 J. E. Guyer and P. W. Voorhees, Phys. Rev. Lett. 74, 4031 (1995); Phys. Rev. B 54, 11710 (1996).

22 B. J. Spencer, P. W. Voorhees, and J. Tersoff, Appl. Phys. Lett. 76, 3022 (2000); Phys. Rev. B 64, 235318 (2001).

23 N. Sridhar, J. M. Rickman, and D. J. Srolovitz, J. Appl. Phys. 82, 4852 (1997).

${ }^{24}$ L. E. Shilkrot, D. J. Srolovitz, and J. Tersoff, Appl. Phys. Lett. 77, 304 (2000); Phys. Rev. B 62, 8397 (2000).

25 J. D. Eshelby, Proc. Roy. Soc. London A 241, 376 (1957).

26 A. A. Maradudin and R. F. Wallis, Surf. Sci. 91, 423 (1980).

27 F. Léonard and R. C. Desai, Phys. Rev. B 57, 4805 (1998); Appl. Phys. Lett. 74, 40 (1999).

28 Z. F. Huang and R. C. Desai, Phys. Rev. B 65, 205419 (2002).

29 B. J. Spencer and J. Tersoff, Phys. Rev. Lett. 79, 4858 (1997); J. Tersoff, Phys. Rev. B 43, 9377 (1991).

${ }^{30}$ P. Sutter and M. G. Lagally, Phys. Rev. Lett. 84, 4637 (2000).

31 R. M. Tromp, F. M. Ross and M. C. Reuter, Phys. Rev. Lett. 84, 4641 (2000).

32 H. Sunamura, N. Usami, Y. Shiraki, and S. Fukatsu, Appl. Phys. Lett. 66, 3024 (1995).

${ }^{33}$ H. Lafontaine, B. F. Mason, S. J. Rolfe, D. D. Perovic, and B. Bahierathan, J. Vac. Sci. Technol. B 16, 599 (1998).

34 A. Ballestad, B. J. Ruck, M. Adamcyk, T. Pinnington, and T. Tiedje, Phys. Rev. Lett. 86, 2377 (2001). 


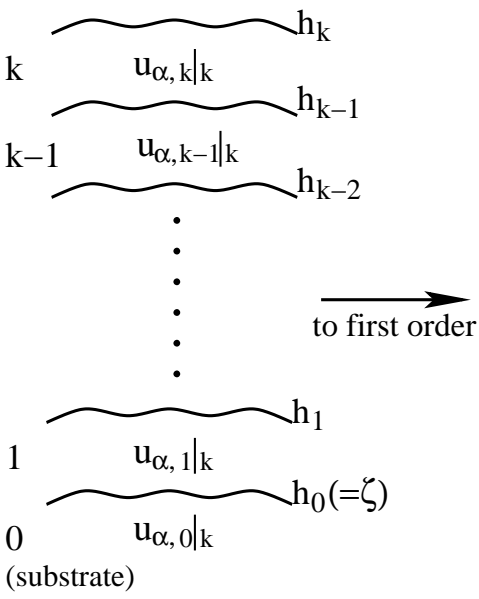

(a)

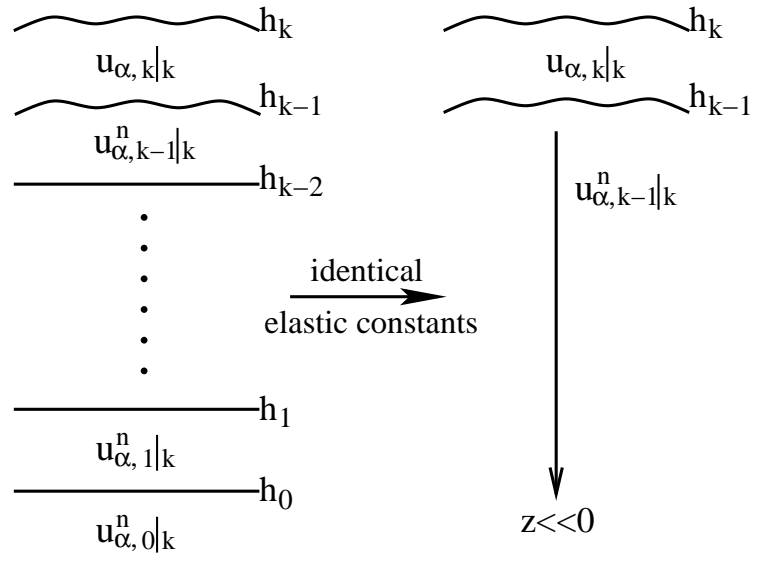

(c)

FIG. 1: Schematic diagram of the simplification procedure for determining the elastic state of the multilayer system. (a) The original $k$-layer structure that we study, with growing and undulating surface (located at $\left.z=h_{k}(x, y, t)\right)$ and with nonplanar and frozen interfaces (located at $z=h_{i}(x, y)$ ) between buried layers $i=k-1, k-2, \ldots, 1$, and 0 (substrate). The elastic displacement field of each layer is indicated as $\left.u_{\alpha, i}\right|_{k}$ with $\alpha=x, y, z$. (b) The effective system of which the elastic state is identical to that of system (a) to first order of perturbations. Note the planar interfaces between layers $i$ and $i-1$ with $0 \leq i \leq k-1$, which are considered to be in the effective elastic media of new-state fields $\left.u_{\alpha, i}^{n}\right|_{k}$. (c) The simplified system that is equivalent to system (b) when the elastic constants of all the layers and substrates are assumed to be identical. Then we have only one layer on a semi-infinite effective medium, with a rough surface and single nonplanar interface. In both (b) and (c), top free surface obeys the boundary condition (16) and the $k /(k-1)$ interface fulfills the special boundary condition (17).

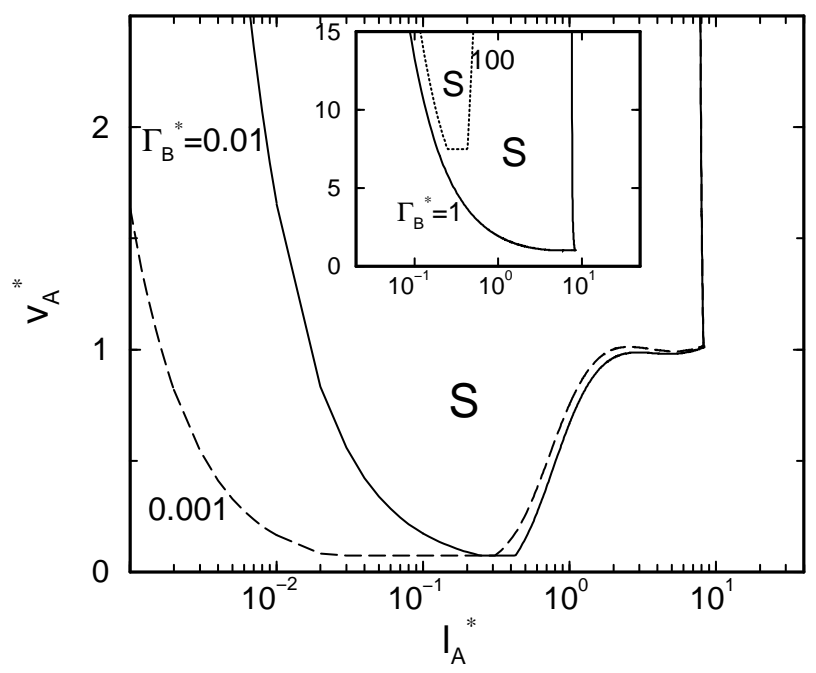

FIG. 2: Stability diagram of rescaled layer thickness $l_{A}^{*}$ versus deposition rate $v_{A}^{*}$, with parameters $\epsilon_{B}^{*}=-1, l_{B}^{*} / l_{A}^{*}=1$, $v_{B}^{*} / v_{A}^{*}=1$, and $\gamma_{B}^{*}=1$ for the growing multilayer system. Different relative surface mobilities $\Gamma_{B}^{*}=0.001$ (dashed curve) and 0.01 (solid curve) are used, as indicated in the figure. Region marked as " $S$ " is effectively stable. Inset: $\Gamma_{B}^{*}=1$ (solid) and 100 (dotted). 

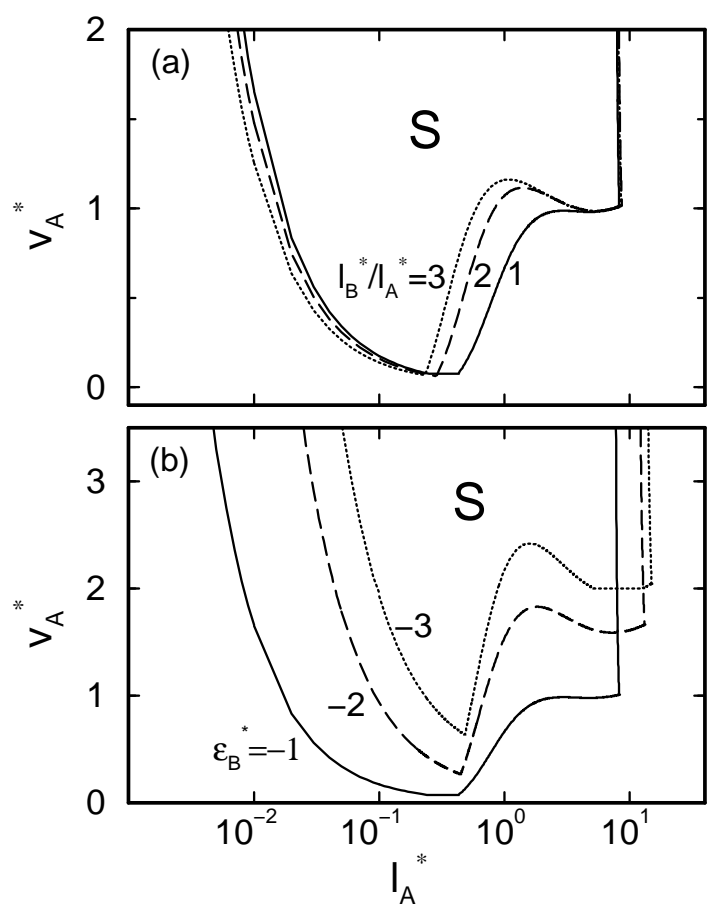

FIG. 3: Stability diagram of $l_{A}^{*}$ versus $v_{A}^{*}$, with parameters $\Gamma_{B}^{*}=0.01, v_{B}^{*} / v_{A}^{*}=1$, and $\gamma_{B}^{*}=1$. (a) $\epsilon_{B}^{*}=-1$, with different values of $l_{B}^{*} / l_{A}^{*}: 1$ (solid), 2 (dashed), and 3 (dotted); (b) Fixed value of $l_{B}^{*} / l_{A}^{*}=1$, but different $\epsilon_{B}^{*}=-1$ (solid), -2 (dashed), and -3 (dotted). 

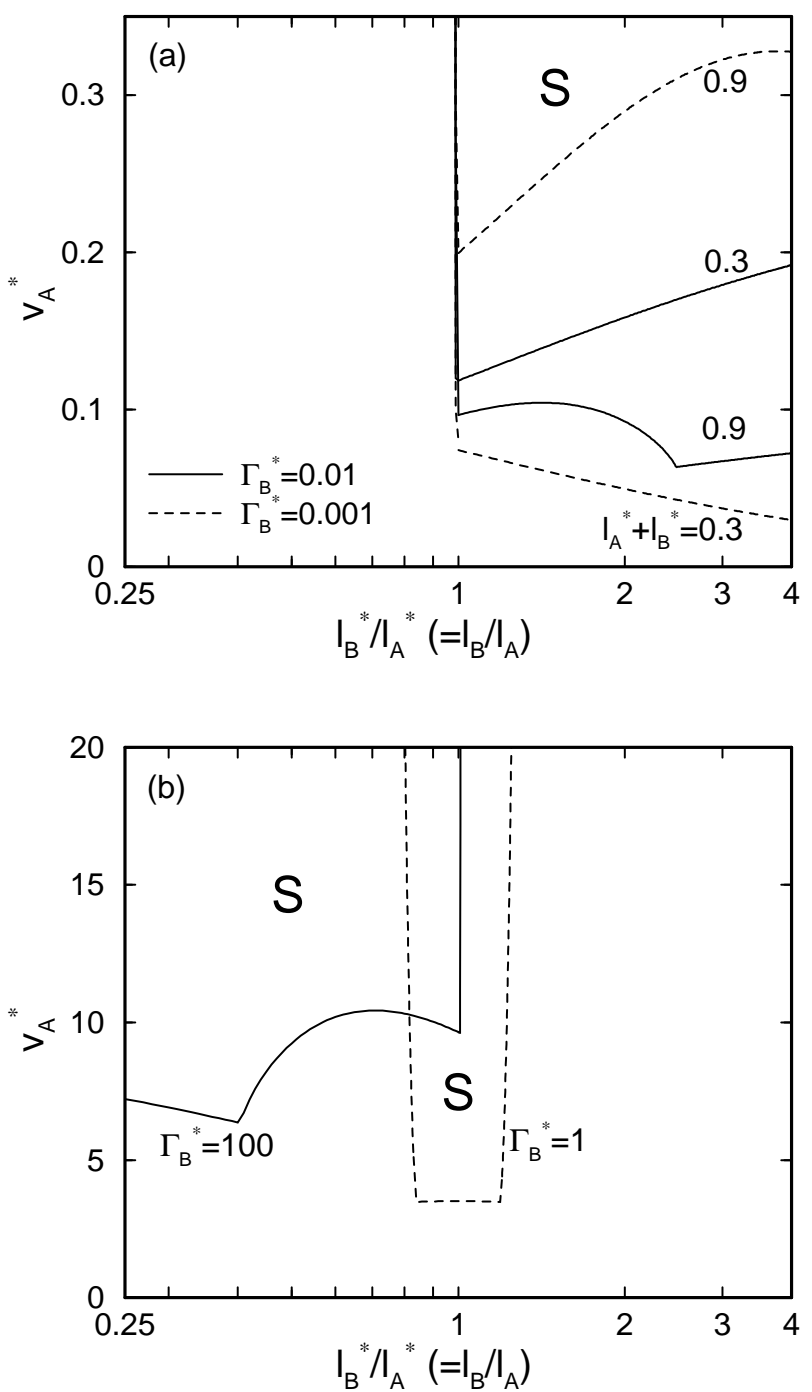

FIG. 4: Stability diagrams of $l_{B}^{*} / l_{A}^{*}$ versus $v_{A}^{*}$, with parameters $\epsilon_{B}^{*}=-1, v_{B}^{*} / v_{A}^{*}=1$, and $\gamma_{B}^{*}=1$. (a) Relative surface mobilities $\Gamma_{B}^{*}=0.01$ (solid) and 0.001 (dashed), with $l_{A}^{*}+l_{B}^{*}=0.9$ and $0.3 ;$ (b) $\Gamma_{B}^{*}=100$ (solid) and 1 (dashed), with $l_{A}^{*}+l_{B}^{*}=0.9$. Different types of asymmetry can be seen for different values of $\Gamma_{B}^{*}$. 

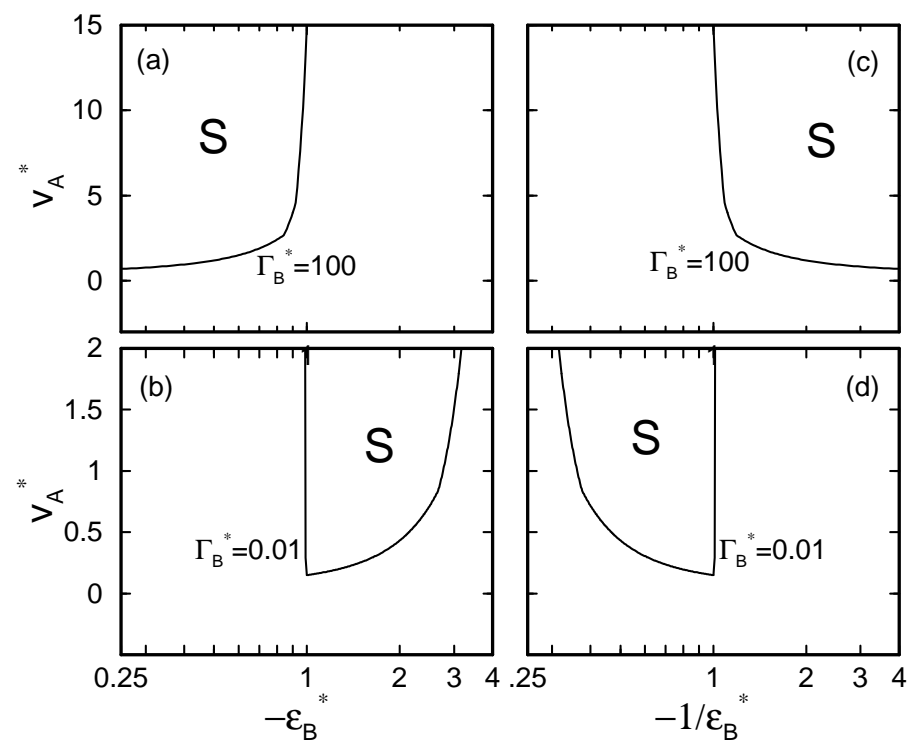

FIG. 5: Stability diagram of misfit $\epsilon_{B}^{*}$ versus $v_{A}^{*}$, with $l_{A}^{*}=l_{B}^{*}=0.5, v_{B}^{*} / v_{A}^{*}=1, \gamma_{B}^{*}=1$, and different $\Gamma_{B}^{*}=100$ (a) and 0.01 (b), showing different types of asymmetry. Panels (c) and (d) are the replots of (a) and (b) as $-1 / \epsilon_{B}^{*}$ versus $v_{A}^{*}$, in order to facilitate comparison respectively with Fig. 6 and its inset, where the strain-balanced condition implies $l_{B}^{*} / l_{A}^{*}=-1 / \epsilon_{B}^{*}$.

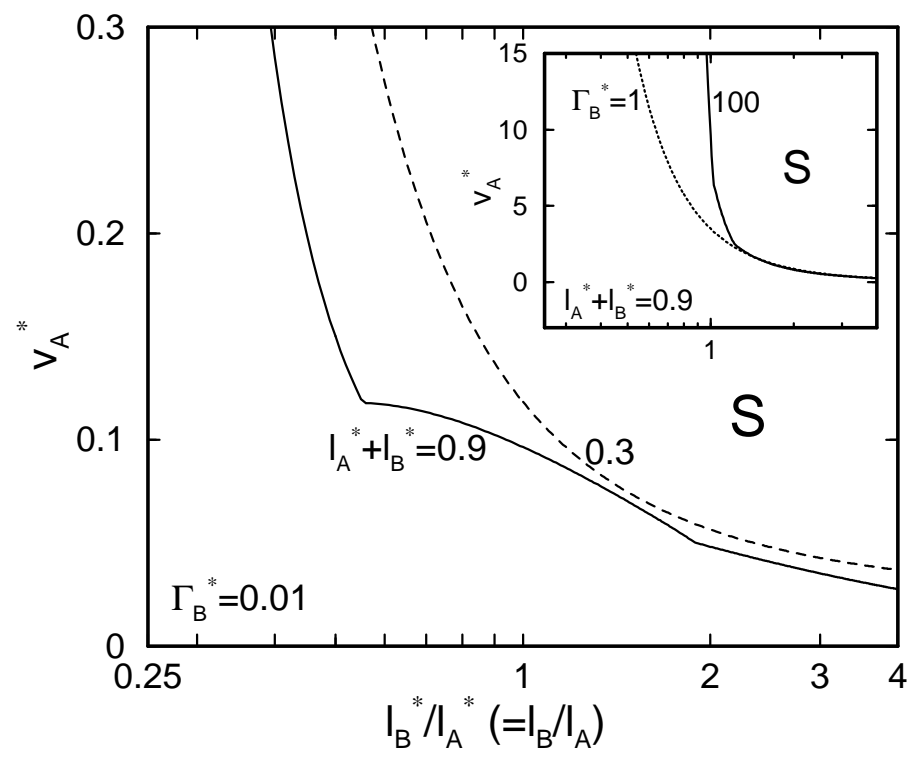

FIG. 6: Stability diagram of $l_{B}^{*} / l_{A}^{*}$ versus $v_{A}^{*}$ in the strain-balanced condition, with $v_{B}^{*} / v_{A}^{*}=1$ and $\gamma_{B}^{*}=1$. For relative mobility $\Gamma_{B}^{*}=0.01, l_{A}^{*}+l_{B}^{*}$ is fixed at 0.9 (solid) and 0.3 (dashed). Inset: $\Gamma_{B}^{*}=100$ (solid) and 1 (dotted), with $l_{A}^{*}+l_{B}^{*}=0.9$. Compared to Figs. 4 and 5, here the stability property shows single type of asymmetry for different $\Gamma_{B}^{*}$. 

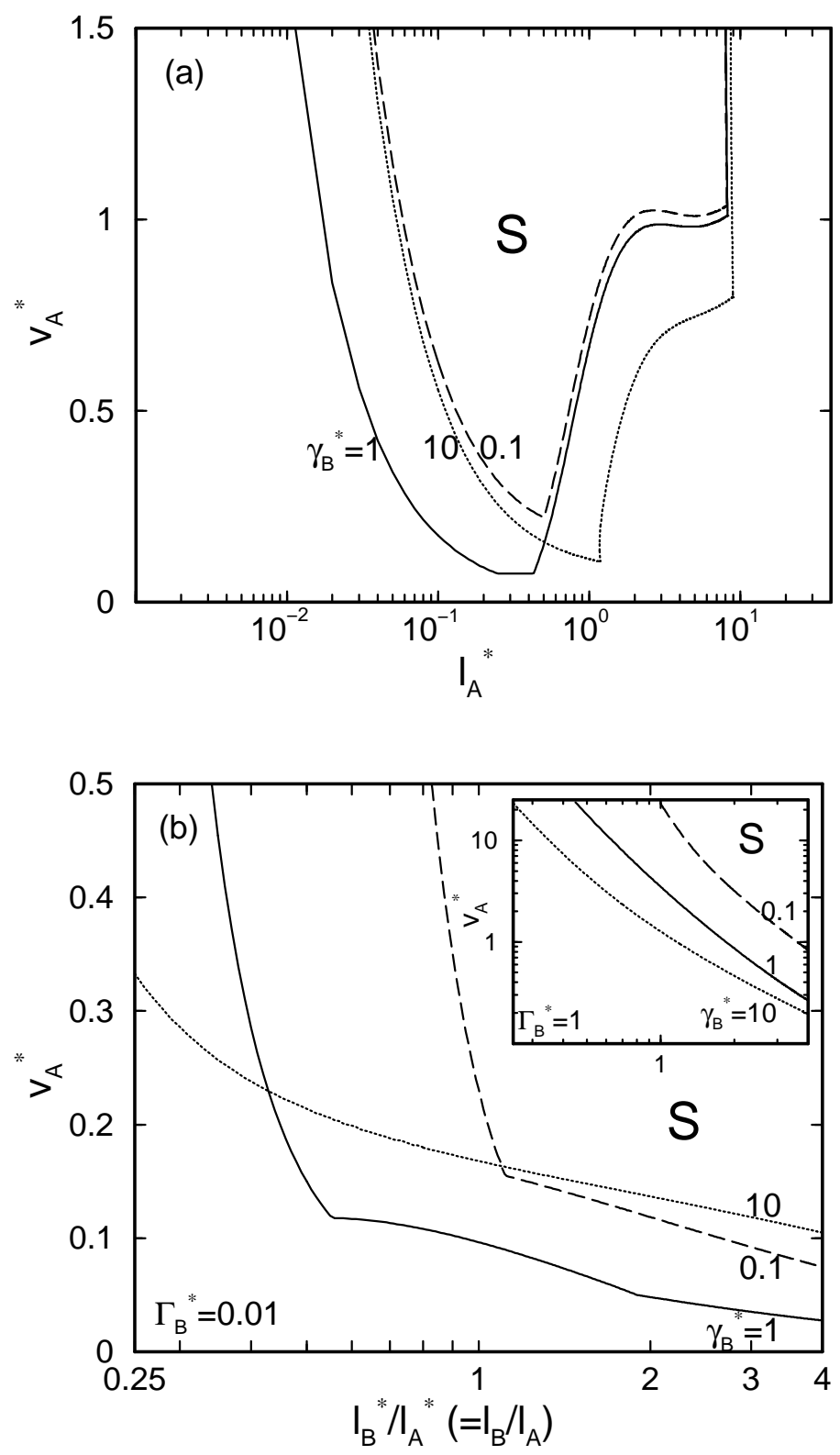

FIG. 7: Stability diagrams for different relative surface tension $\gamma_{B}^{*}=0.1$ (dashed), 1 (solid), and 10 (dotted), with $v_{B}^{*} / v_{A}^{*}=1$. In (a), $v_{A}^{*}$ is plotted as a function of $l_{A}^{*}$, with $\Gamma_{B}^{*}=0.01, \epsilon_{B}^{*}=-1, l_{B}^{*} / l_{A}^{*}=1$, and $v_{B}^{*} / v_{A}^{*}=1$; while in (b), the system is in the strain-balanced condition, and we use different $\Gamma_{B}^{*}\left[0.01\right.$ and 1 (inset)] in the stability diagram of $l_{B}^{*} / l_{A}^{*}$ versus $v_{A}^{*}$, with fixed $l_{A}^{*}+l_{B}^{*}=0.9$. 


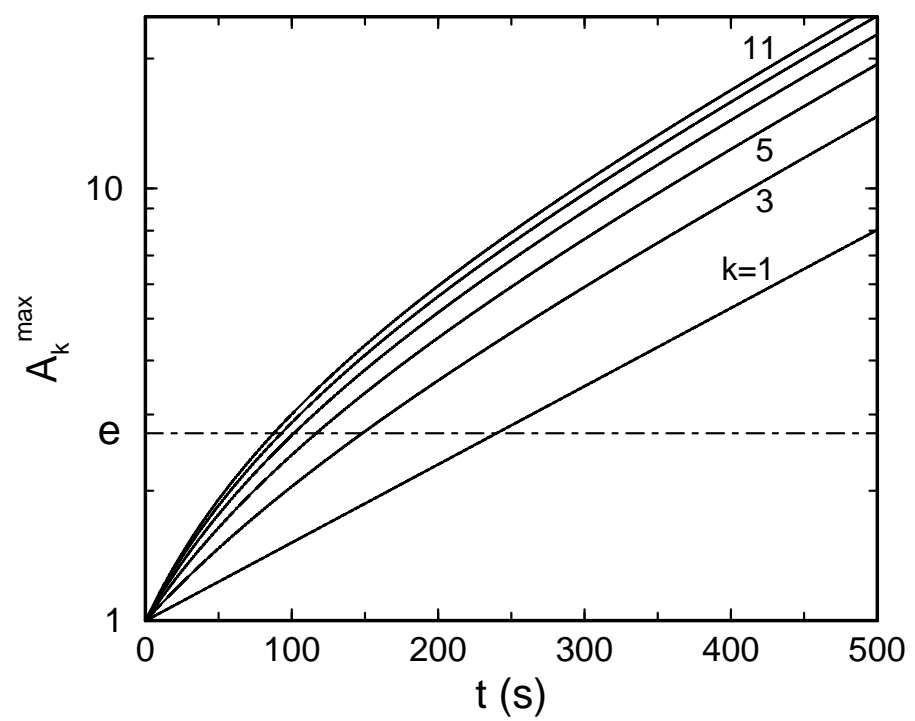

FIG. 8: Maximum perturbation amplitude $A_{k}^{\max }$ versus time $t$, for successive strained layer numbers $k=1,3, \ldots, 11$ (from bottom to top). Note the semilog plot. The material parameters used are analogous to those of Ge/Si(001) growth system, with $l_{A}=4 \mathrm{ML}, l_{B}=9 \mathrm{~nm}, v_{A}=1 \mathrm{ML} / \mathrm{min}$, and $T=550{ }^{\circ} \mathrm{C}$.

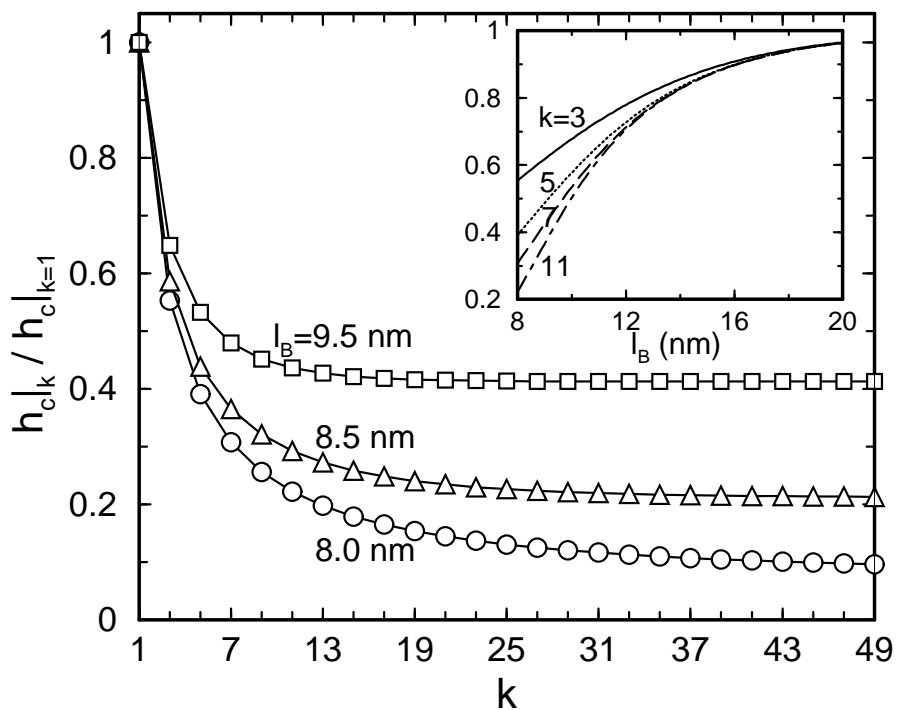

FIG. 9: Kinetic critical thickness $\left.h_{c}\right|_{k}$ (rescaled by that of single layer $\left.h_{c}\right|_{k=1}$ ) as a function of the layer number $k$, with the $\mathrm{Ge} / \mathrm{Si}(001)$ growth parameters: $l_{A}=4 \mathrm{ML}, v_{A}=1 \mathrm{ML} / \mathrm{min}, T=550{ }^{\circ} \mathrm{C}$, and different spacer layer thickness $l_{B}=9.5 \mathrm{~nm}$ (square), $8.5 \mathrm{~nm}$ (triangle), and $8 \mathrm{~nm}$ (circle). Inset: $\left.h_{c}\right|_{k} /\left.h_{c}\right|_{k=1}$ as a function of $l_{B}$, for $k=3,5,7$, and 11 . 


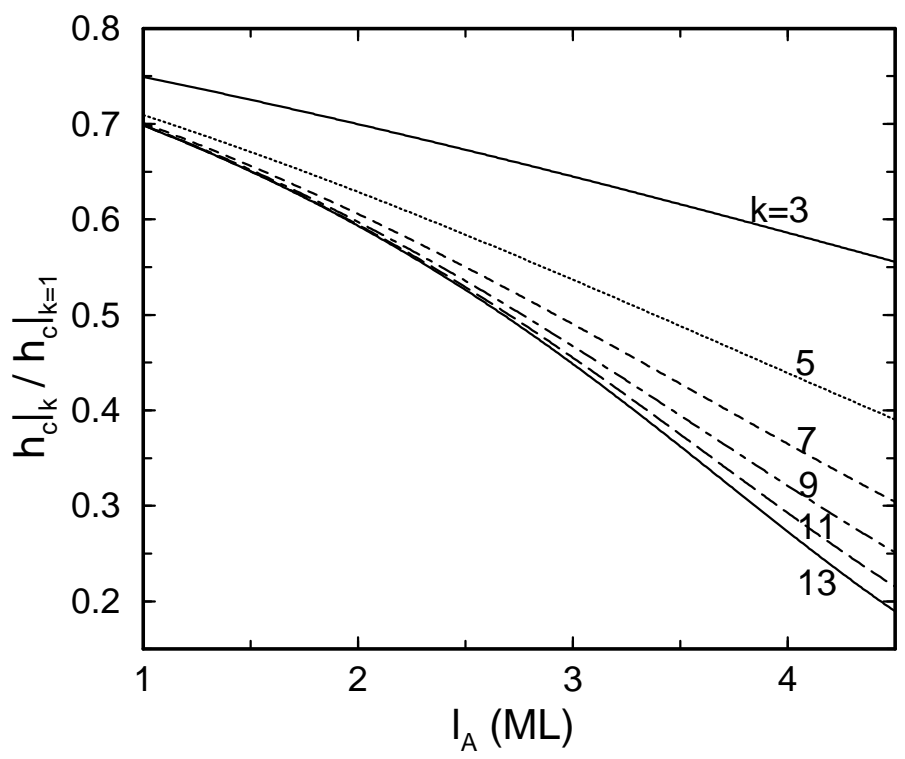

FIG. 10: Rescaled kinetic critical thickness $\left.h_{c}\right|_{k} /\left.h_{c}\right|_{k=1}$ as a function of average strained layer thickness $l_{A}$, for successive strained layer numbers. $l_{B}=8.5 \mathrm{~nm}, v_{A}=1 \mathrm{ML} / \mathrm{min}$, and $T=550{ }^{\circ} \mathrm{C}$. 

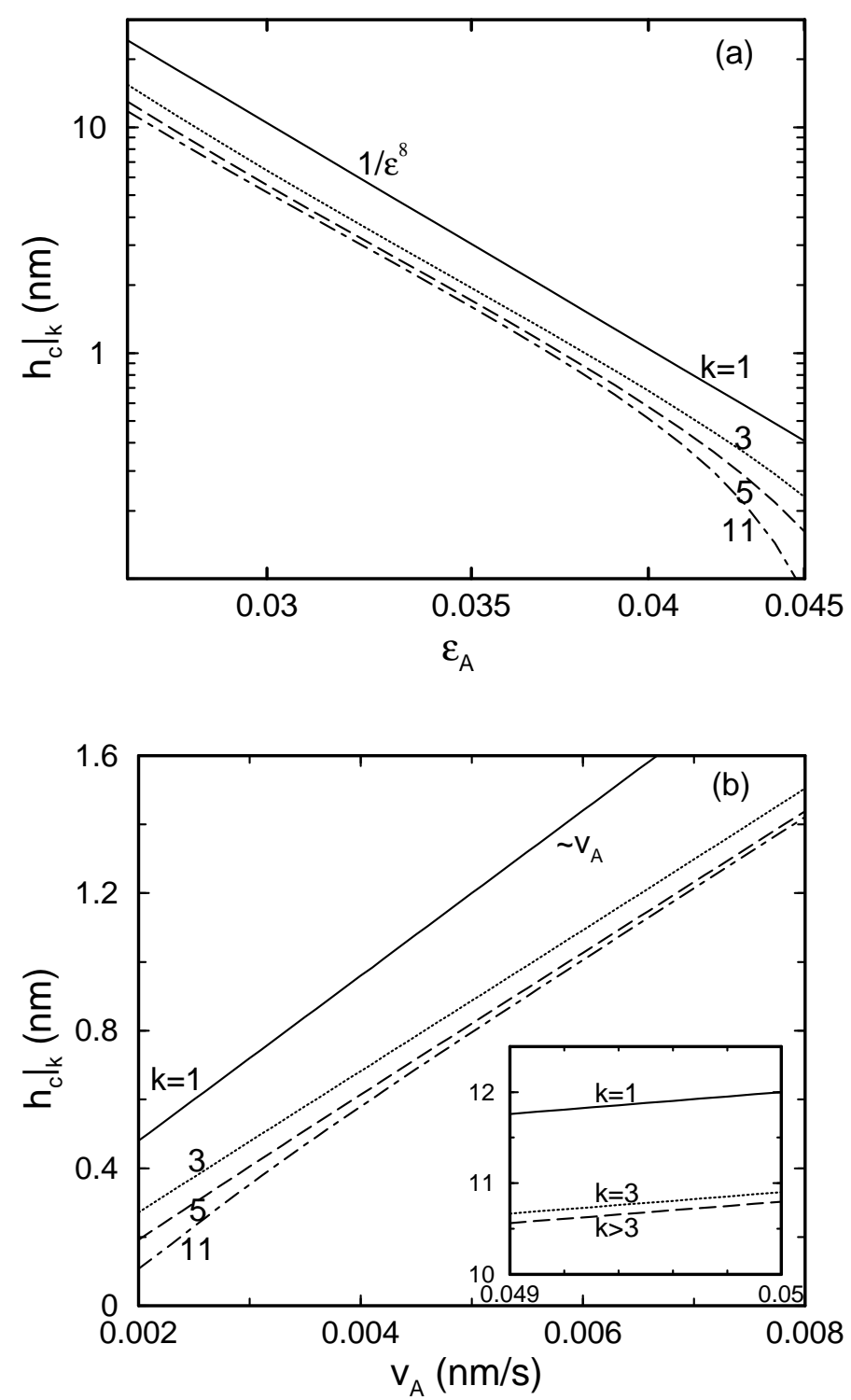

FIG. 11: Kinetic critical thickness $\left.h_{c}\right|_{k}$ as a function of (a) misfit $\epsilon_{A}$ and (b) deposition rate $v_{A}$, for different layer numbers $k=1,3,5$, and 11 . The parameters are $l_{A}=4 \mathrm{ML}, l_{B}=9 \mathrm{~nm}$, and $T=550{ }^{\circ} \mathrm{C}$ for both (a) and (b), as well as $v_{A}=1$ $\mathrm{ML} / \mathrm{min}$ for (a). Note the inset in (b) where the range of $v_{A}$ is almost ten times larger. 